\title{
Revocatoria de mandato subnacional en ciudades grandes de América del Sur: Lima Metropolitana (2013) en perspectiva comparada $^{+}$
}

\author{
MARIANA RAMÍREZ BUSTAMANTE* \\ Pontificia Universidad Católica del Perú \\ ramirezb.mariana@pucp.pe
}

https://doi.org/10.18800/rcpg.201602.008

\section{RESUMEN}

A pesar de que la revocatoria del mandato ha sido históricamente un mecanismo poco utilizado y bastante cuestionado, en las últimas décadas se ha expandido en América Latina. Dicha expansión se debe a su introducción en las leyes y a frecuentes activaciones o intentos de activación del mecanismo. En esta línea, el objetivo del presente artículo es identificar las condiciones institucionales, sociales y políticas que inciden en el éxito o fracaso de estos intentos de revocatoria de autoridades locales. Con dicha finalidad, se analizan las experiencias de revocatoria en las grandes ciudades de América del Sur.

Palabras clave: revocatoria de mandato, revocatoria local, ciudades grandes, Sudamérica, Lima Metropolitana.

Revocation of subnational mandate in large cities of South America: Metropolitan Lima (2013) in comparative perspective

\section{Abstract}

Though historically recall referendum mechanism has been the least used, and the most controversial, in the last decades it has expanded in Latin America. This expansion is caused by its inclusion in laws and frequent activations or attempts to activate this direct democracy mechanism (DDM). The aim of this paper is to identify the institutional, social and political conditions that have an impact in the success or failure of the attempts to use this mechanism, by analyzing the experiences in large cities in South America with this DDM.

Key words: recall referendum, recall referendum at a local level, large cities, South America, Metropolitan Lima.

\footnotetext{
* Máster en Estudios Latinoamericanos por la Universidad de Salamanca y Licenciada en Ciencia Política y Gobierno por la Pontificia Universidad Católica del Perú (PUCP). Miembro del Grupo de Investigación de Política Subnacional (GIPS) de la PUCP y pre-docente de la misma casa de estudios.

+ Recibido el 4 de enero de 2017; aceptado el 27 de febrero de 2017.
} 



\section{INTRODUCCIÓN}

Los retos que presenta la democracia contemporánea, tales como los elevados niveles de insatisfacción y desconfianza hacia los partidos políticos y, en general, la apatía hacia el sistema democrático han propiciado reformas a favor de una democracia más participativa en distintos países (Altman, 2011; Dalton, Burklin y Drummond, 2001; Whitehead, 2011). En efecto, «la búsqueda de formas de representación política más auténticas y que unan con más cercanía a los gobernantes con los ciudadanos, es un tema global» (Whitehead, 2011, p. 16). Así, en las últimas décadas, se han incluido instituciones de participación directa popular, las cuales «dan voz a grupos que usualmente no son escuchados en las elecciones» (Cameron, Hershberg y Sharpe, 2012, p. 1), independientemente de la ideología política de los partidos o de los gobiernos (Ramírez y Welp, 2011), las cuales están modificando la democracia representativa — «con su dependencia a las elecciones, partidos y policy making exclusivamente por los poderes legislativos y ejecutivos» (Cameron et al., 2012, p. 5)—, aunque no la socavan.

Entre las nuevas formas de participación política se encuentran los mecanismos de democracia directa (MDD). Estos son instituciones por medio de las cuales los ciudadanos pueden decidir o emitir opinión de manera directa sobre diversos asuntos a través del sufragio universal y secreto (Altman, 2010). Uno de estos mecanismos es la revocatoria de mandato, que es «susceptible de ser activada por la ciudadanía, para consultar en un referéndum vinculante sobre la interrupción del mandato de una autoridad electa» (Welp y Serdült, 2014, p. 2). Al respecto, es importante destacar dos aspectos. Por un lado, la revocatoria es el MDD menos extendido a nivel internacional y cuenta con tradición de uso en pocos lugares (Welp y Serdült, 2017). De otro lado, se registra un aumento de solicitudes de revocatoria — pese a que no siempre se logran activar las consultas- y existen más posturas a favor de introducir esta institución en otros países (Welp y Serdült, 2011).

Si bien existe una extensa literatura sobre los MDD, es mucho más reducido el interés académico en el mecanismo de revocatoria de mandato (Welp, 2014, 2015), puesto que una reducida cantidad de países cuentan con normativas que lo incluyen durante un periodo de tiempo suficiente para su implementación y, a su vez, por la diferencia en las sendas que siguen estos países (Serdült y Welp, 2016). En consecuencia, los estudios sobre la revocatoria son más recientes y, con algunas excepciones (Welp, 2015, 2014, 2013; Welp y Serdült, 2011, 2012, 2017; Eberhardt, 2013), se caracterizan por ser estudios descriptivos. Por lo general, se han desarrollado análisis de un único caso de 
estudio $^{1}$, donde se resalta el contexto de incorporación de la revocatoria de mandato, el diseño institucional, su puesta en práctica y los efectos singulares de estas experiencias. Empero, hasta el momento son escasos los análisis comparativos que exploren los factores que inciden en el intento de revocatoria de mandato y expliquen la reducida cantidad de experiencias que consiguen activar la consulta ${ }^{2}$.

Algunos estudios señalan que la mayor proporción de revocatorias son activadas — se realiza la consulta popular de revocatoria en contra de la(s) autoridad(es), tras cumplir con los requisitos establecidos por ley- en municipios pequeńos, facilitado por una población reducida (Tuesta, 2014a; Welp, 2014; Welp y Serdült, 2012), y/o en circunscripciones donde existen elevados niveles de competencia política, donde por lo general los promotores de la revocatoria son los rivales que han perdido frente al elegido (Franco-Cuervo, 2014a). Ello se debe a que en municipios con menor población resulta más sencillo recabar el número de firmas y seguir los procedimientos necesarios para activar la revocatoria, o «que los partidos políticos de la oposición sean los mejor situados para hacerlo» (Welp y Serdült, 2012, p. 190); de tal forma que las razones profundas parecen vincularse más a la lucha por el poder que a una mejora de la calidad institucional.

En esta línea, el objetivo del presente estudio es contribuir a una mejor comprensión de las condiciones que explican la efectividad por activar los procesos de revocatoria; entendida como la manifestación directa por parte de la ciudadanía por medio de la votación sobre la interrupción del mandato de la autoridad sometida a consulta, independientemente de si esta es revocada o no. Para ello se analizan el total de experiencias de revocatoria registradas desde el ańo de la primera aplicación hasta 2015, en ciudades grandes de Sudamérica de más de 1 millón de habitantes, con la finalidad de realizar un estudio comparado. En ese sentido, primero se identifican las ciudades grandes que han registrado intentos de revocatoria en la subregión y, en segundo lugar, se determinan los factores que influyen sobre la efectividad de la activación, considerando a) el diseńo institucional, b) el apoyo a la autoridad y las causas explícitas para la activación del mecanismo, y c) el rol de los actores políticos.

El artículo se estructura de la siguiente manera. Primero, se presenta el estado del arte y la metodología que será utilizada. Luego, se presentan los intentos

\footnotetext{
Existen estudios de caso sobre Argentina (Arques, 2014a, 2014b), Bolivia (Verdugo, 2014, 2014a), Costa Rica (Rivera, 2006), Cuba (Guzmán Hernández, 2014), Ecuador (Castellanos Santamaría, 2014; Ramírez, 2014), Estados Unidos (Qvortrup, 2014), Suiza (Serdült, 2014); Perú (Tuesta, 2014a, 2014b; Welp, 2013; ONPE, 2013a; Del Águila, 2013; Remy, 2005, 2008, 2013; Wiener, 2004, entre otros), Colombia (Jiménez, 2001; Bustos, 2002; Martínez Cárdenas, 2013; Franco-Cuervo, 2014a, 2014b) y Venezuela (Kornblith, 2014a, 2014b, 2009, 2007).

2 Con la excepción del estudio de Uribe (2016), que analiza la revocatoria municipal en Colombia.
} 
formales de revocatoria en ciudades grandes de América del Sur; el diseño institucional que se aplica para la activación de este MDD, los casos que lograron alcanzar los requisitos; así como el origen y las causas de los mismos. Posteriormente, se realiza un análisis del proceso de revocatoria de Lima Metropolitana (2013). Finalmente, se presentan las conclusiones y reflexiones finales.

\section{Aproximaciones Sobre el meCanismo de revocatoria en América LATINA}

La figura de la revocatoria se contempla en ocho países de América Latina, principalmente en la región andina - Venezuela, Colombia, Ecuador, Perú y Bolivia-, al igual que en algunos estados mexicanos y provincias argentinas ${ }^{3}, \mathrm{y}$ en Panamá, aunque únicamente para autoridades nacionales (véase Cuadro 1). Este instrumento se ha vuelto popular en la región debido a la debilidad de los mecanismos de rendición de cuentas (Welp y Serdült, 2014, p. 114), con un uso más extendido a escala subnacional. En efecto, la mayoría de revocatorias han tenido lugar en municipios pequeńos, facilitado por una población reducida $^{4}$; así como por relaciones políticas y sociales más cercanas, donde es más probable la personificación de la política (Battle, 2014, p. 97) y donde suele haber una tendencia más marcada hacia la construcción de redes clientelistas por parte de líderes locales (Moncada, 2011³; Benton, 2012, p. 254).

La literatura existente da cuenta de tres condiciones que parecen influir sobre las posibilidades de activar la revocatoria de mandato. En primer lugar, debido a que la activación de este MDD implica contar con una organización y recursos tales como tiempo, recursos humanos y materiales (Serdült y Welp, 2012), los requisitos más laxos podrían incentivar a un actor o a un grupo de actores a intentar revocar a una autoridad, y viceversa (Tuesta, 2014b; Welp, 2013). Asimismo, se argumenta que el uso de la revocatoria puede explicarse por la combinación del diseño institucional del proceso, es decir por las reglas para activar este MDD y su posibilidad de éxito o de aplicarse efectivamente; así como por el nivel de institucionalización del sistema de partidos (Welp, 2015).

\footnotetext{
3 En Cuba también se incorporaron mecanismos de participación ciudadana, como la revocatoria, tras el triunfo de la revolución (1959) y una vez consolidado el gobierno de partido único, por medio de la Constitución de 1976. No obstante, paulatinamente, este mecanismo ha caído en desuso (Guzmán Hernández, 2014). Sin embargo, no se consideró como un mecanismo de democracia directa, al no contar con un régimen democrático.

4 La revocatoria es más utilizada a escala local, al igual que otros MDD, como el referéndum, «debido a que son más fáciles de organizar localmente» (Scarrow, 2001, p. 660).

5 Como explica Moncada (2011, p. 4), «el clientelismo está anclado a lazos sociales y políticos a nivel micro y las redes son difíciles de organizar y explorar a escala nacional».
} 
Cuadro 1. Países que incluyen el mecanismo de revocatoria de mandato

\begin{tabular}{|c|c|c|}
\hline Autoridades que pueden ser revocadas & Países & Cantidad \\
\hline Todas las autoridades elegidas popularmente & Ecuador, Venezuela, Bolivia & 3 \\
\hline Autoridades nacionales (a) & $\begin{array}{c}\text { Panamá, Rusia, Etiopía, Kirguistán, } \\
\text { Nigeria, Liberia, Uganda }\end{array}$ & 7 \\
\hline Autoridades subnacionales & $\begin{array}{c}\text { Colombia (b), Perú, Japón, Polonia, } \\
\text { Columbia Británica (Canadá) (a), Filipinas, } \\
\text { algunos lander de Alemania (b) }\end{array}$ & 7 \\
\hline Órgano colectivo en su conjunto & Suiza (6 cantones) & 1 \\
\hline Varía según estado o provincia & Argentina, México, Estados Unidos & 3 \\
\hline Total & 21 & \\
\hline
\end{tabular}

Fuente: Elaboración propia, sobre la base de Welp (2014, p. 27; 2013, p. 54).

(a) Solo autoridades legislativas.

(b) Solo autoridades ejecutivas.

En segundo lugar, algunos autores destacan que el mecanismo de revocatoria puede configurarse como un «instrumento de defensa de la ciudadanía frente a gobernantes devenidos impopulares» (Lissidini, 2007, p. 4), asegurando la rendición de cuentas por parte de las autoridades (Smulovitz, 2001). De esta forma, la revocatoria representa un método de accountability vertical, donde los ciudadanos no se vean limitados a esperar nuevas elecciones para castigar o premiar al mandatario o a su partido, sino que pueden hacerlo antes de que culmine su periodo (Welp, 2015; Verdugo, 2014; Breuer, 2007).

Por último, múltiples estudios demuestran que la revocatoria de mandato es utilizada por ex autoridades o perdedores de elecciones como una herramienta política (Franco-Cuervo, 2014a, 2014b; Tuesta, 2014a, 2014b; Welp, 2014; ONPE, 2013b; Remy, 2013, 2008, 2005; Wiener, 2004, entre otros), en busca de desplazar de sus cargos a los ganadores; tal como evidencian claramente los casos de Perú y Colombia. Empero, al dirigirse a personas y no a asuntos, como una política o ley (Kaufmann, Büchi y Bran, 2008; Kaufmann y Waters, 2004; Papadopoulous, 1995), algunos consideran que la revocatoria «se activa contra una institución de democracia representativa» (Altman, 2011, p. 16); configurándose, más bien, como una herramienta de «perpetuación electoral» (Del Águila, 2013, p. 3). Más aún, recientes estudios de caso demuestran que la activación del mecanismo de revocatoria suele ser causado por escándalos, por el impacto de los medios digitales - los cuales permiten una mayor rapidez y alcance de la campaña-, sumado al liderazgo de los partidos políticos en el proceso de activación del referéndum revocatorio en contra de sus opositores (Welp y Serdült, 2017). 


\section{Metodología}

El presente estudio se centra en el análisis de las causas de la efectividad del mecanismo de revocatoria en ciudades con más de 1 millón de habitantes donde se registran intentos de activación de la revocatoria de mandato a escala local en Argentina, Bolivia, Colombia, Ecuador, Perú y Venezuela; puesto que no se encontró evidencia de su utilización en México y en Panamá, se incluye este MDD únicamente para autoridades nacionales. En cada país se analizaron los periodos durante los cuales se encontró vigente la ley para activar dicho MDD a nivel subnacional. Al respecto, cabe señalar que en Venezuela no se contó con legislación específica sobre MDD; sin embargo el proceso de revocatoria de autoridades regionales y locales de 2007 se llevó a cabo tras una simplificación de la regulación y la definición de normas por parte del Consejo Nacional Electoral (Kornblith, 2014b). No se encontró evidencia de la utilización de este MDD a nivel local en Venezuela en otra ocasión, razón por la cual únicamente se consideró el proceso de 2007.

Asimismo, cabe precisar que se tomó en cuenta el criterio del tamańo de la ciudad - a partir de 1 millón de habitantes_- en tanto el ámbito urbano es el que concentra la mayor población en la región latinoamericana, la cual representa aproximadamente el $80 \%$ de la población (Cadena et al., 2011), con lo cual se observa la dinámica política de mayor alcance y de gran importancia. Se consideró dicho criterio para acotar la selección de los casos de estudio debido a la gran proporción de revocatorias en municipios pequeños, facilitada por una población reducida; sumado al hecho de que el análisis y la sistematización de las consultas llevadas a cabo enfrentan la limitación de acceder a información detallada sobre los contextos en los que estas prácticas se han producido, sobre todo a nivel local.

Por efectividad de la revocatoria se entiende a la manifestación directa de la voluntad popular por medio de la votación ciudadana en las urnas, a través de la cual se define la interrupción del mandato de la autoridad sometida a consulta antes de finalizar su mandato; independientemente de que se le revoque o no. Por el contrario, se considera como inefectivo cuando, a pesar de cumplir con los requisitos establecidos por la normativa para presentar la solicitud formal de revocatoria de la autoridad, no se logra la activación de la misma; es decir, no se realiza la consulta popular, por medio de la cual los ciudadanos se manifiesten a favor o en contra de la revocatoria. Se asume esta definición de efectividad, ya que esta implica que la revocatoria cumple su razón de ser: de dotar a la ciudadanía de voz y voto en el proceso de destitución de la autoridad cuestionada (Welp y Serdült, 2012). 
Esta investigación se centra en la efectividad de este MDD dado que, pese al aumento de solicitudes de revocatoria en contra de autoridades locales en la región, en la mayoría de los casos no se logra activar la consulta popular. En efecto, al comparar las solicitudes formales presentadas ante el organismo electoral correspondiente para iniciar el proceso de revocatoria a nivel local, se observa que tan solo en los casos de Argentina y Colombia se activó el 20\% de las solicitudes de revocatoria presentadas (véase Cuadro 2).

Cuadro 2. Intentos de revocatoria vs. procesos activados a escala subnacional

\begin{tabular}{|c|c|c|c|c|c|c|}
\hline \multirow[b]{2}{*}{ País } & \multirow[b]{2}{*}{ Periodo } & \multirow{2}{*}{$\begin{array}{l}\text { Solicitudes } \\
\text { presentadas }\end{array}$} & \multicolumn{2}{|c|}{ Revocatorias activadas } & \multirow{2}{*}{$\begin{array}{c}\text { Porcentaje de } \\
\text { activación } \\
\text { local } \\
(\%)\end{array}$} & \multirow[b]{2}{*}{ Fuente } \\
\hline & & & Provincial & $\begin{array}{l}\text { Distrital/ } \\
\text { Local }\end{array}$ & & \\
\hline Argentina & $2002-2015$ & 10 & - & 2 & $20 \%$ & $\begin{array}{l}\text { Arques (2014a); } \\
\text { Welp y Serdült } \\
\quad(2017)^{*}\end{array}$ \\
\hline Bolivia & $\begin{array}{c}\text { Mediados } \\
\text { de 2012- } \\
2013\end{array}$ & 124 & - & 0 & $0 \%$ & $\begin{array}{l}\text { Verdugo (2014); } \\
\text { Welp y Serdült } \\
\quad(2017)^{*}\end{array}$ \\
\hline Colombia & $1991-2014$ & 169 & - & 34 & $20,12 \%$ & $\begin{array}{l}\text { Welp (2016; 2015); } \\
\text { Franco-Cuervo } \\
\text { (2014a); RNEC } \\
\text { de la República de } \\
\text { Colombia* }\end{array}$ \\
\hline Ecuador & $2010-2015$ & 801 & - & 78 & $9,74 \%$ & $\begin{array}{l}\text { Ramírez }(2014, \\
\text { pp. 141-145)* }\end{array}$ \\
\hline Perú & $1997-2013$ & 5539 & 11 & 1018 & $18,38 \%$ & $\begin{array}{c}\text { ONPE }(2013 a, \\
\text { pp. } 31,40)\end{array}$ \\
\hline Venezuela & 2007 & 167 & - & 10 & $5,99 \%$ & $\begin{array}{l}\text { Kornbilth (2014b, } \\
\text { p. 239); Welp (2015) }\end{array}$ \\
\hline
\end{tabular}

Fuente: Elaboración propia sobre la base de revisión bibliográfica, datos oficiales de instituciones electorales y medios de prensa escrita en versión digital sobre solicitudes formales de revocatoria de mandato presentadas.

* Datos actualizados en función de la revisión de los medios de prensa escrita.

En Bolivia no se logró activar ninguna de las solicitudes de revocatoria presentadas durante el periodo 2012-2013. Por su parte, en Argentina se activaron 2 procesos de las 10 solicitudes presentadas entre 2002 y 2015. En Colombia se registraron 130 iniciativas de revocatoria de mandato de alcaldes, durante el periodo 1996-2012 (MOE, 2012), y en 2013 hubo 39 presuntos intentos de revocatoria (Franco-Cuervo, 2014b, p. 105). Por su parte, hasta el 
año 2010, se presentaron 730 peticiones de recolección de firmas para activar revocatorias de mandato en Ecuador, según el CNE. Después de la reforma de 2011, se registraron otras 54 solicitudes de revocatoria, pero ninguna superó el proceso de admisión; a las cuales se sumaron 17 intentos más (Mestanza, 2015). En Perú, la cifra de intentos de revocatoria se basa en la cantidad de kits electorales vendidos por la Oficina Nacional de Procesos Electorales (ONPE); según la cual se han presentado más de 5500 solicitudes para activar dicho MDD entre 1997 y 2013. De otro lado, en el proceso de revocatoria de autoridades regionales y locales de 2007 de Venezuela se presentaron 167 solicitudes de revocatoria, de las cuales únicamente se activaron 10.

Así pues, el presente estudio plantea como hipótesis que el mecanismo de revocatoria será efectivo si el diseño institucional es menos exigente para su activación por parte de la ciudadanía, si existe un nivel elevado de desaprobación ciudadana de la gestión del edil, y si la consulta es promovida por una organización política con capacidad suficiente para cumplir con los requisitos para activar el proceso de revocatoria, lo cual difícilmente podría lograr una organización ciudadana.

El diseño institucional de la revocatoria se refiere a los acuerdos establecidos a través de las normas, de los cuales dependen qué tan flexibles son los requisitos para activar dicho mecanismo por parte de los ciudadanos. Al respecto, se sistematizó información sobre el periodo durante el cual se puede activar la revocatoria ( $\mathrm{Pr})$, las causas y/o justificaciones que se requieren presentar $(\mathrm{C})$, el porcentaje de firmas que debe recopilarse $(\mathrm{F})$ y el periodo de tiempo para recabar las firmas (Pf) en los casos analizados, por medio de la revisión de la normativa sobre la materia. A partir de estas dimensiones, se construyó un índice de dificultad para la activación de la revocatoria de mandato a escala local; asignando un puntaje de menor a mayor, en función de la exigencia de los requisitos. Con la excepción del indicador de causas (C) — que por lo general no es necesario-, se optó por agregar las demás dimensiones a través de una fórmula multiplicativa, dado que ninguno compensa la ausencia del otro; por el contrario, se trata de condiciones necesarias y suficientes para la activación de la revocatoria de mandato.

\section{Indice de dificultad para la activación de la revocatoria de mandato a escala local}

$$
I=\left[\operatorname{Pr}^{*} F^{*} 2 P f\right]+C
$$

Se le otorgó un puntaje doble al periodo para recopilar firmas, puesto que, a pesar de que el porcentaje de firmas sea reducido, si el plazo establecido es 
limitado, será sumamente complicado cumplir con la cantidad necesaria ${ }^{6}$. Se recategorizó la escala del índice, de tal forma que va del 100 al 0; donde 100 es el nivel de dificultad más alto y el 0 el más bajo. Se calcularon tres rangos a partir de la desviación estándar $(30,02)$ y la media aritmética $(20,43)^{7}$; de tal forma que i) de 50,46 a más se consideró como un nivel de dificultad alto, ii) de 20,43 a 50,45 se consideró como un nivel de dificultad medio, y iii) menor a 20,42 se consideró como un nivel de dificultad bajo.

De otro lado, el nivel de desaprobación ciudadana hace referencia al respaldo político hacia el alcalde sometido a consulta por parte de la ciudadanía; lo cual se medirá a partir del porcentaje de aprobación y desaprobación ciudadana hacia la gestión municipal. Este aspecto se abordará a partir de encuestas de opinión pública y noticias publicadas en medios de prensa escritos.

El origen de la promoción de la consulta ciudadana se refiere a los actores que intervinieron en la activación de la iniciativa de revocatoria. Siguiendo a Serdült y Welp (2012, p. 74), se pueden presentar tres escenarios:

i. Ciudadanos como tomadores de decisiones, donde la democracia directa funcionara desde abajo (bottom-up) para distribuir el poder en democracias representativas donde la ciudadanía o las organizaciones de la sociedad civil juegan un rol en la activación de este MDD.

ii. Partidos políticos u opositores políticos como principales jugadores, donde la utilización de MDD constituye una estrategia de los partidos para movilizar electores — en potencia- o para establecer asuntos en la agenda pública.

iii. Estrategia del gobierno — tanto local como nacional — para aumentar su poder, cuando utilizan el MDD como una herramienta para controlar el gobierno.

En el presente estudio se definió el grupo que promueve la solicitud de revocatoria — ciudadanos u organizaciones de la sociedad civil, opositores políticos del alcalde o partido de gobierno nacional — a partir de la revisión del

\footnotetext{
6 Para definir el nivel de dificultad para la activación del mecanismo de revocatoria en los países o municipios analizados se consideró el periodo de activación, donde se considera que «después del primer año» es el menos exigente, por lo que se le asignó el valor de 1, mientras que el más exigente es «luego de la mitad del mandato y antes del último año" al que se le dio el valor de 6 . Asimismo, se asignó el valor de 0 a los casos donde no se solicita ninguna causa y 2 cuando se establece más de un motivo específico para la activación del MDD. Se otorgó el valor de 6 al porcentaje más elevado del porcentaje de firmas — del 40\%— y 1 al más bajo —-menor al 7\% del padrón electoral—. Se le dio el valor de 0 si no se especifica el plazo para recabar las firmas y 6 al plazo más reducido — de 3 días_-, a los cuales se le asignó un peso doble por ser el requisito más difícil de cumplir.

7 El primer rango se definió a partir del promedio menos 1 desviación estándar, hasta la media aritmética (-1a a 0); el segundo, a partir del promedio, hasta la media aritmética más 1 desviación estándar $(0$ a $1 \alpha)$, y el tercero, a partir del promedio más 1 desviación estándar, hasta la media aritmética más 2 desviaciones estándar (1 a a $2 \alpha$ ).
} 
proceso de recolección de firmas para iniciar el proceso, por medio de revisión bibliográfica y de prensa escrita.

\section{ANÁLISIS}

América del Sur, y particularmente la región andina, constituyen las subregiones donde se ha utilizado de manera más intensa el mecanismo de revocatoria de mandato (Welp, 2015). No obstante, el uso de este MDD en ciudades grandes resulta bastante limitado y en aquellos casos donde se ha promovido su utilización, por lo general se ha dado en las ciudades capitales. En efecto, con la excepción del caso de Argentina, la proporción de intentos de revocatoria de autoridades subnacionales en ciudades con más de 1 millón de habitantes fue inferior al 2\% del total presentado (véase Cuadro 3).

Cuadro 3. Porcentaje de solicitudes de revocatoria en ciudades grandes ( $\geq 1$ millón de habitantes) de América del Sur (\%)

\begin{tabular}{|c|c|c|c|c|c|}
\hline País & $\begin{array}{c}\text { Ciudades grandes } \\
\text { ( } \geq 1 \text { millón de habitantes) }\end{array}$ & $\begin{array}{l}\text { Solicitudes } \\
\text { de } \\
\text { revocatoria }\end{array}$ & $\begin{array}{l}\text { Intentos } \\
\text { en ciudades } \\
\text { grandes }\end{array}$ & $\begin{array}{l}\mathrm{N}^{\circ} \text { de } \\
\text { intentos en } \\
\text { ciudades } \\
\text { grandes }\end{array}$ & $\begin{array}{l}\text { Porcentaje } \\
\text { de } \\
\text { intentos }\end{array}$ \\
\hline Argentina & $\begin{array}{l}\text { Ciudad de Buenos Aires } \\
\qquad(3028 \text { 481) }\end{array}$ & 10 & $\begin{array}{c}\text { Córdoba (2002), } \\
\text { Buenos Aires } \\
(2005,2014)\end{array}$ & 3 & 30,00 \\
\hline Bolivia & Santa Cruz (1 523 488) (b) & 124 & -- & -- & 0,00 \\
\hline Colombia & $\begin{array}{c}\text { Medellín (2 } 219 \text { 861) } \\
\text { Barranquilla (1 } 112 \text { 889) } \\
\text { Bogotá, D.C (6 } 6778 \text { 691) } \\
\text { Cali }(2075 \text { 380) (c) }\end{array}$ & 169 & $\begin{array}{l}\text { Bogotá (1999, } \\
\text { 2013) }\end{array}$ & 2 & 1,18 \\
\hline Ecuador & $\begin{array}{l}\text { Guayaquil (2 } 440553) \\
\text { Quito (2 } 319671)(d)\end{array}$ & 801 & Quito (2010) & 1 & 0,12 \\
\hline Perú & Lima $(7622792)(e)$ & 5539 & Lima (2013) & 1 & 0,02 \\
\hline Venezuela & Distrito capital (2 073 055) (f) & 167 & Caracas (2007) & 1 & 0,60 \\
\hline
\end{tabular}

Fuente: Elaboración propia sobre la base de revisión bibliográfica, de datos oficiales en instituciones electorales y en medios de prensa escrita, vía virtual.
(a) Estimaciones de población por sexo, departamento y ańo calendario 2010-2025. o38, Serie análisis demográfico del Instituto Nacional de Estadística y Censos de la República Argentina (2015).
(b) En base a datos del Censo de 2012 del Instituto Nacional de Estadística del Estado Plurinacional de Bolivia.
(c) En base a datos del Censo de 2005 del Departamento Administrativo de Estadística de la República de Colombia.
(d) En base a datos del Censo de 2010 del Instituto Nacional de Estadística y Censos de la República de Ecuador.
(e) En base a datos del Censo de 2007 del Instituto Nacional de Estadística e Informática del Perú.
(f) En base a datos del Censo de 2011 del Instituto Nacional de Estadística de la República de Venezuela. 
En Argentina se presentaron dos intentos de revocatoria en la capital, Buenos Aires (2005 y 2014), y en la ciudad de Córdoba (2002). En Colombia, la mayoría de alcaldes de la capital han sido objeto de intentos de revocatoria con poco éxito desde 1994 (Franco-Cuervo, 2014a, p. 67); así, Bogotá registra dos intentos formales de revocatoria (1999 y 2013). Por su parte, en Ecuador, Venezuela y Perú se presentaron una solicitud de revocatoria en Quito (2010), Caracas (2007) y Lima (2013), respectivamente. Bolivia fue el único país de la subregión que no registró ninguna solicitud de revocatoria en una ciudad grande.

De las ocho solicitudes de revocatoria en ciudades grandes, tan solo dos alcanzaron los requisitos exigidos por ley. Únicamente se aprobaron los procesos de revocatoria de Lima (2013), en contra de la alcaldesa Susana Villarán y todos los miembros del Concejo municipal, y de Bogotá (2013), en contra del alcalde Gustavo Petro (véase Cuadro 4).

Cuadro 4. Solicitudes de revocatoria y procesos activados en ciudades grandes $(\geq 1$ millón de habitantes) de América del Sur

\begin{tabular}{|c|c|c|c|c|c|}
\hline País & Ciudad & Habitantes & Impulsado por & Resultado & Fuente \\
\hline \multirow{3}{*}{ Argentina } & $\begin{array}{l}\text { Córdoba } \\
(2002)\end{array}$ & 1329604 & Ciudadanía & $\begin{array}{l}\text { Junta de firmas } \\
\text { inconclusa y } \\
\text { cancelación } \\
\text { del proceso de } \\
\text { revocatoria }\end{array}$ & $\begin{array}{l}\text { Arques (2014a, } \\
\text { p. 170) }\end{array}$ \\
\hline & $\begin{array}{c}\text { Buenos Aires } \\
\text { (2005) }\end{array}$ & 2776138 (a) & Alcalde & $\begin{array}{l}\text { Junta de firmas } \\
\text { inconclusa }\end{array}$ & $\begin{array}{c}\text { Schneider y Welp } \\
(2015 \text {, p. 31)/ Arques } \\
\text { (2014a, p. 162) }\end{array}$ \\
\hline & $\begin{array}{c}\text { Buenos Aires } \\
\text { (2014) }\end{array}$ & 3049229 (b) & $\begin{array}{l}\text { Ciudadanía/ } \\
\text { opositores } \\
\text { políticos }\end{array}$ & No prosperó & $\begin{array}{l}\text { Schneider y Welp } \\
(2015, \text { p. 31)* }\end{array}$ \\
\hline \multirow[b]{2}{*}{ Colombia } & $\begin{array}{l}\text { Bogotá } \\
(1999)\end{array}$ & 1606004 (c) & $\begin{array}{l}\text { Excandidato } \\
\text { y opositores } \\
\text { políticos }\end{array}$ & $\begin{array}{l}\text { Junta de firmas } \\
\text { inconclusa }\end{array}$ & $\begin{array}{c}\text { Franco-Cuervo } \\
\text { (2014a, pp. 67-68)* }\end{array}$ \\
\hline & $\begin{array}{l}\text { Bogotá } \\
(2013)\end{array}$ & 2385391 (d) & $\begin{array}{c}\text { Congresista } \\
\text { del Partido } \\
\text { de gobierno - } \\
\text { Partido de la } \\
\text { U y opositores } \\
\text { políticos }\end{array}$ & $\begin{array}{c}\text { Cancelación } \\
\text { del proceso de } \\
\text { revocatoria por } \\
\text { judicialización del } \\
\text { mismo }\end{array}$ & $\begin{array}{c}\text { Franco-Cuervo } \\
\text { (2014a, pp. 62-65) }\end{array}$ \\
\hline Ecuador & $\begin{array}{l}\text { Quito } \\
(2010)\end{array}$ & 2319671 (e) & Ciudadanía & $\begin{array}{l}\text { No se admitió } \\
\text { recolección de } \\
\text { firmas }\end{array}$ & $\begin{array}{l}\text { Schneider y Welp } \\
(2015, \text { p. 32)* }\end{array}$ \\
\hline
\end{tabular}




\begin{tabular}{|c|c|c|c|c|c|}
\hline País & Ciudad & Habitantes & Impulsado por & Resultado & Fuente \\
\hline Perú & Lima (2013) & $8617314(\mathrm{f})$ & $\begin{array}{c}\text { Político de } \\
\text { partido de } \\
\text { exalcalde } \\
\text { de Lima } \\
\text { Metropolitana }\end{array}$ & $\begin{array}{c}\text { No se revocó a la } \\
\text { alcaldesa, pero sí a } \\
\text { 22 de un total de } \\
\text { 39 regidores (g) }\end{array}$ & $\begin{array}{c}\text { ONPE (2014a); } \\
\text { Oruna Vásquez } \\
(2014,2014 \mathrm{a})\end{array}$ \\
\hline Venezuela & $\begin{array}{c}\text { Caracas } \\
(2007)\end{array}$ & $2064913(\mathrm{~h})$ & No se especifica & $\begin{array}{c}\text { No se logra recabar } \\
\text { el porcentaje de } \\
\text { firmas necesario }\end{array}$ & $\begin{array}{c}\text { Kornblith (2014b, } \\
\text { p. 238) }\end{array}$ \\
\hline
\end{tabular}

Fuente: Elaboración propia sobre la base de revisión bibliográfica, de datos oficiales en instituciones electorales y en medios de prensa escrita, vía virtual.
(a) Población correspondiente al año 2001, según el censo realizado en ese año del INDEC.
(b) Población correspondiente al año 2014, según proyecciones por departamento del INDEC.
(c) Población correspondiente al ańo 1999, según «Estimaciones 1985-2005 y proyecciones 2005-2020 de hogares nacional y departamental por área», del Departamento Administrativo Nacional de Estadística - DANE.
(d) Población correspondiente al año 2013, según «Estimaciones 1985-2005 y proyecciones 2005-2020 de hogares nacional y departamental por área", del DANE.
(e) Proyección de la población ecuatoriana, por años calendarios según cantones, del Instituto Nacional de Estadística y Censos de Ecuador; para 2010.
(f) Población correspondiente al ańo 2013, según Población Estimada y Proyectada por Departamento y Provincia, 2000-2015, del INEI.
(g) En la revocatoria de Lima Metropolitana de 2013 se destituyó a más de un tercio de las autoridades del Concejo Municipal de la Municipalidad Metropolitana de Lima.
(h) Proyección de población al 30 de junio con base al censo 2011, según parroquias y sexo, 2000-2050, del Instituto Nacional de Estadística de la República Bolivariana de Venezuela.
* Datos actualizados en función de la revisión de los medios de prensa escrita.

\section{a. Diseño institucional en los intentos de revocatoria}

La mayoría de los intentos de revocatoria se vieron frustrados al no presentar la cantidad de firmas necesarias para la aprobación de la solicitud. En Bogotá (1999), Córdoba (2002), Buenos Aires $(2005,2014)$ y Caracas (2007) no se logró concluir la recolección de la cantidad de firmas necesarias; aunque solo en los dos últimos se señala un plazo específico para cumplir con dicho requisito. Cabe destacar que en el primer intento formal de revocatoria en Bogotá (1999), la Registraduría Nacional del Estado Civil (RNEC) —institución responsable de la consulta - determinó que las firmas no cumplían los requisitos para la activación de los procesos de revocatoria (Rey, 2015); debido a la anulación de más del $60 \%$ de las rúbricas presentadas. De otro lado, en el caso de Quito (2010), el Consejo Nacional Electoral (CNE) negó la entrega del formato para la recolección de firmas, por falta de una formulación clara y precisa del motivo por el cual se intentaba revocar al alcalde y al vicealcalde (Schneider y Welp, 2015, p. 33).

\footnotetext{
8 Se anularon 421 mil firmas del total de 672 mil presentadas ante la RNEC; con lo cual no se cumplió con el requisito de 507 mil firmas (Redacción El Tiempo, 1999a).
} 
En la línea anterior, pareciera que la reducida cantidad de procesos de revocatoria activados se explica por el diseńo institucional de este MDD. Sin embargo, al realizar el cálculo del índice de dificultad de activación de este MDD, se observa que tan solo dos de los trece casos presentan niveles altos de dificultad. Actualmente, la normativa más exigente es la boliviana (2010), lo cual podría explicar la no activación de este MDD en ningún municipio con más de 1 millón de habitantes; seguido por Venezuela (2007). Este diseño institucional de Bolivia establece que únicamente se puede solicitar la revocatoria luego de la mitad del mandato de la autoridad y antes del último año, con el $25 \%$ del censo a nivel departamental y regional, el $20 \%$ a nivel provincial y el $30 \%$ a nivel municipal, firmas que deben ser recabadas en un plazo máximo de noventa días. El marco normativo de Venezuela establece que se puede solicitar una revocatoria a partir de la mitad del mandato y se requiere presentar el 20\% del padrón electoral, cifra que debe ser recabada en tres días.

Por su parte, la normativa vigente de Ecuador (2010) y el anterior diseño institucional venezolano (2003) registran niveles intermedios de dificultad para activar la revocatoria. En Ecuador, el porcentaje de firmas necesarios varía entre el 25\% —en circunscripciones que cuenten, como máximo, con $5 \mathrm{mil}$ electores - y el 10\% —en circunscripciones con más de 300 mil electores-, proceso que solo se puede iniciar después del primer año de gobierno y antes del último, por actos de corrupción o incumplimiento del plan de trabajo. De otro lado, la anterior norma venezolana (2003) tan solo difiere de la actual en tanto se contaba con un día más para recopilar la cantidad de firmas (se pasó de cuatro a tres días, con la normativa de 2007).

Así pues, el resto de normativas presentan niveles de dificultad baja para la activación de la revocatoria de mandato: Colombia (2002, 1994), Ciudad de Buenos Aires (2000), Ecuador (1998) y, sobre todo, Bolivia (2009), Ecuador (2008), Ciudad de Córdoba (2002), Venezuela (1999) y Perú (1994) (véase Cuadro 6).

Con la normativa colombiana de 1994, permitió solicitar la revocatoria de mandato únicamente en casos de insatisfacción general de la ciudadanía o por incumplimiento del plan de gobierno y estableció como requisito recabar el $40 \%$ del padrón electoral, luego del primer año del gobierno. Con la modificación de 2002, se agregó un plazo de 180 días para la recolección de la cantidad de firmas necesarias, con lo cual aumentó ligeramente el nivel de exigencia.

De otro lado, en Buenos Aires (2000) es posible solicitar la revocatoria de una autoridad local luego del primer año de mandato y antes de los seis meses, alegando causas atenientes al desempeño de sus funciones, con el $20 \%$ del 
censo electoral, el cual debe ser recabado en un periodo de doce meses. La normativa ecuatoriana de 1998 era menos exigente, ya que permitía solicitar la revocatoria de una autoridad luego del primer año de mandato y antes del último, únicamente por actos de corrupción o por un incumplimiento injustificado del plan de trabajo, con la presentación del 30\% del censo electoral que puede ser recabado en un plazo indefinido. Posteriormente, con la reforma de 2008, se disminuyó el nivel de exigencia, al no ser necesario especificar el motivo de la solicitud de revocatoria de la autoridad y al solicitar el 10\% del censo electoral.

Al igual que la normativa ecuatoriana de 2008, Córdoba (2002), Venezuela (1999) y Perú (1994) registraron los niveles más bajos de dificultad. En la ciudad de Córdoba se permite presentar una solicitud formal tras el primer año de mandato y antes de los últimos nueve meses sin especificar las causas, con el apoyo del 10\% de firmas del censo electoral recabadas en un plazo indeterminado. Con el marco normativo venezolano de 1999 tampoco se solicitaba presentar justificación para iniciar una revocatoria de mandato ni un plazo para la recolección del $20 \%$ de las firmas del padrón electoral de la circunscripción de la autoridad en cuestión. A diferencia de la normativa peruana, no se establecía un máximo de firmas y se determinaba que podía activarse la revocatoria luego de la mitad del periodo elegido.

Del mismo modo, el diseńo institucional de este MDD en Perú (1994) — anterior a la reforma de 2015- permitía iniciar la solicitud tras el primer año de gobierno y antes del último sin necesidad de presentar causas, sin determinar un plazo límite para recabar el 25\% de firmas del padrón electoral. Más aún, en Lima — que cuenta con una población mayor a 9 millones de habitantes - se necesitaba presentar una cantidad de firmas sumamente reducida ya que el marco legal establecía un máximo de 400 mil firmas, equivalente al 6,7\% de los electores (Tuesta, 2014b, p. 49), siendo la única circunscripción donde se aplicaba. Así pues, durante el periodo 1997-2013, se han presentado más de 5 mil intentos de revocatoria en Perú; de los cuales más de cuatro mil quinientos (4505) buscaron iniciar procesos de revocatoria a autoridades distritales (ONPE, 2013a; Welp, 2013), principalmente en municipios pequeños, con menos de 5 mil electores, usualmente de la Sierra, predominantemente rurales y pobres (Tuesta 2014a, pp. 18-19; ONPE, 2013b, p. 69). De esta forma, Perú se ha convertido no solo en el país con la mayor cantidad de procesos de revocatoria en América Latina (Del Águila, 2013: 3), sino también en el mundo (Tuesta, 2014b, p. 20). 


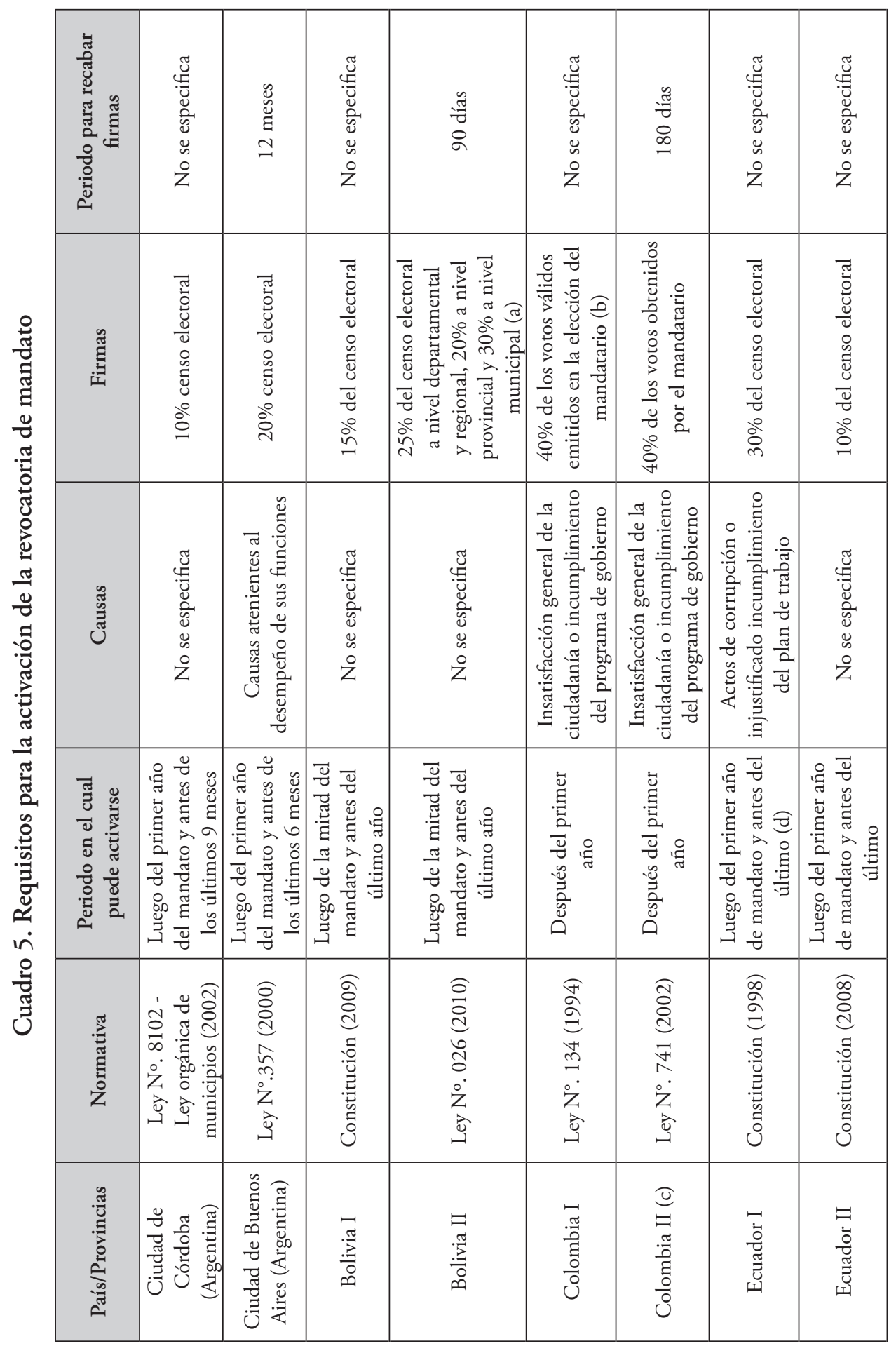




\begin{tabular}{|c|c|c|c|c|c|}
\hline 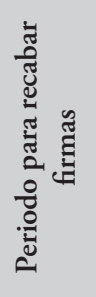 & 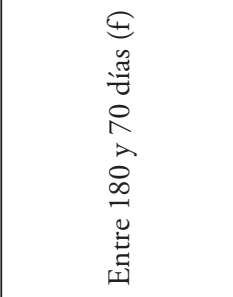 & 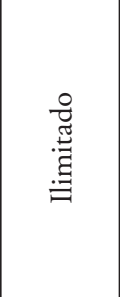 & 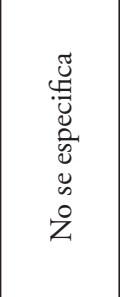 & $\frac{\mathscr{g}}{\stackrel{\mathscr{J}}{ت}}$ & $\frac{\mathscr{g}}{\tilde{g}}$ \\
\hline 菉 & 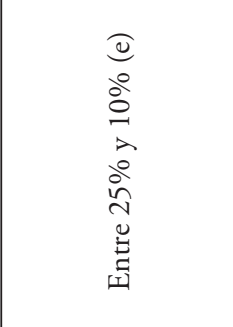 & 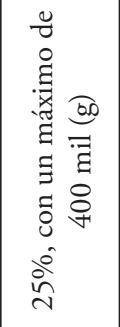 & 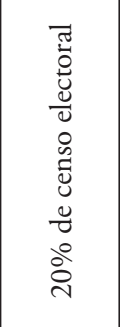 & 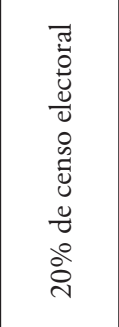 & 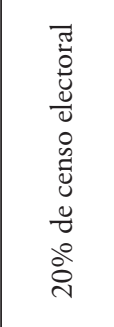 \\
\hline 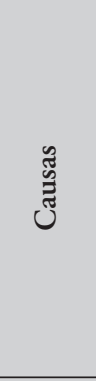 & 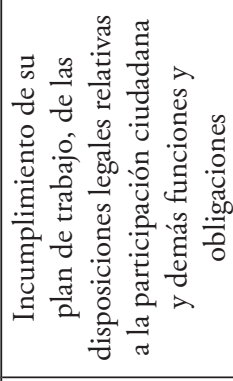 & 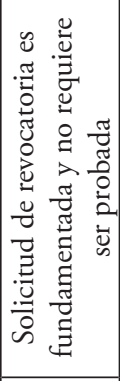 & 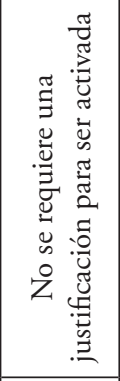 & 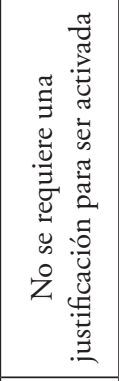 & 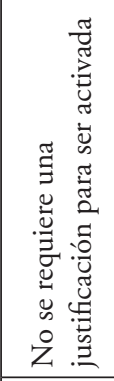 \\
\hline 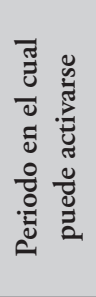 & 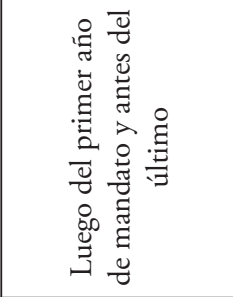 & 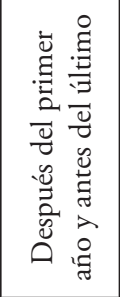 & 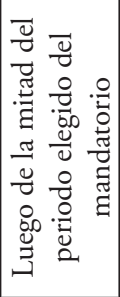 & 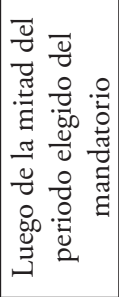 & 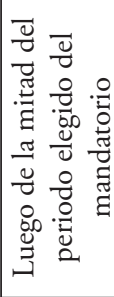 \\
\hline 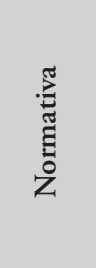 & 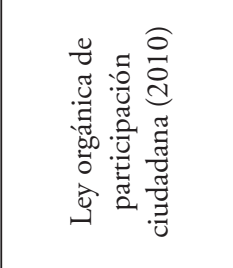 & 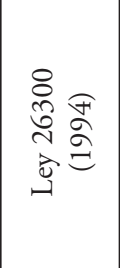 & 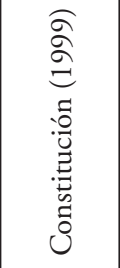 & 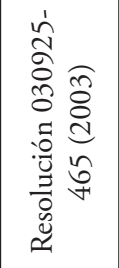 & 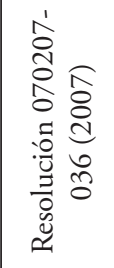 \\
\hline 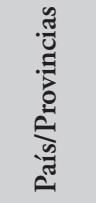 & 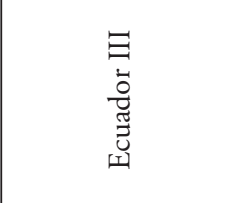 & $\bar{\Xi}$ & 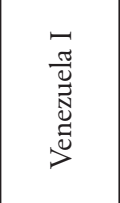 & 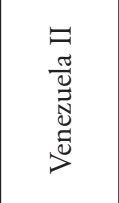 & 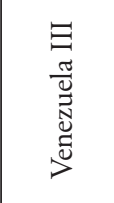 \\
\hline
\end{tabular}

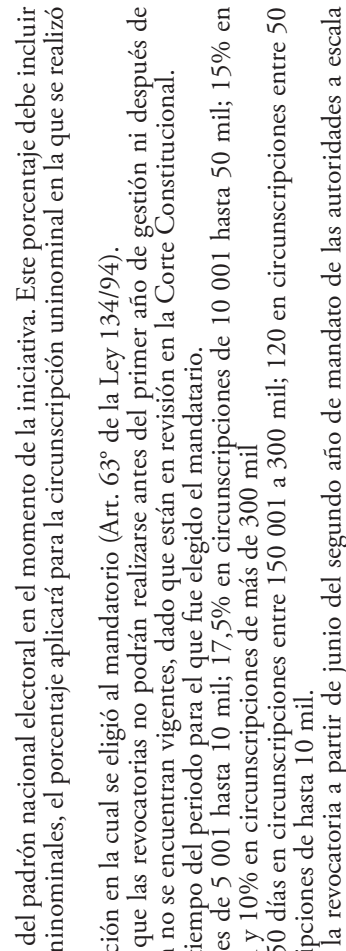

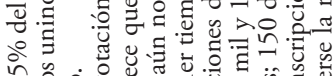
却。

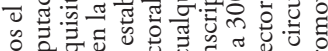

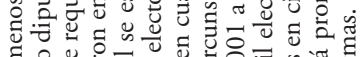
过

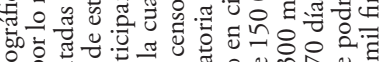
응.

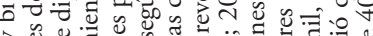
入

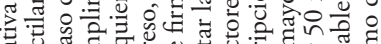

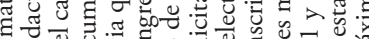

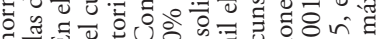

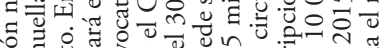

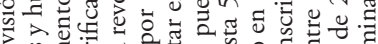

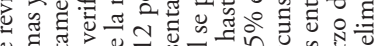

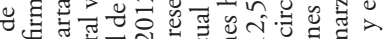

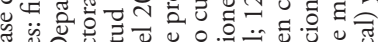

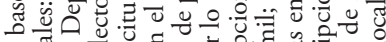

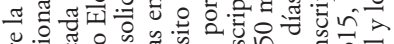

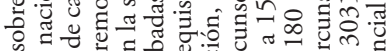

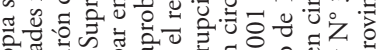

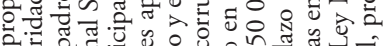
ㄴ.

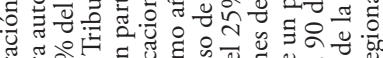

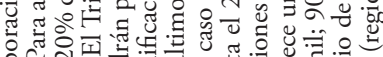

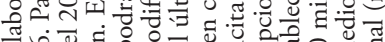

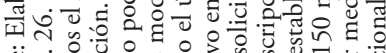

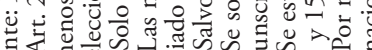

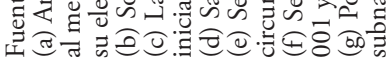


Cuadro 6. Índice de dificultad de activar el mecanismo de revocatoria municipal

\begin{tabular}{|c|c|c|c|c|c|c|c|}
\hline Nivel de dificultad & País & $\begin{array}{l}\text { Caso al } \\
\text { que se } \\
\text { aplica }\end{array}$ & $\begin{array}{c}\text { Periodo } \\
\text { para } \\
\text { activarse }\end{array}$ & Causas & Firmas & $\begin{array}{l}\text { Periodo } \\
\text { para } \\
\text { recabar } \\
\text { firmas }\end{array}$ & Índice \\
\hline \multirow{2}{*}{$\begin{array}{l}\text { Dificultad alta } \\
\text { (50,46 a más) }\end{array}$} & $\begin{array}{c}\text { Bolivia II, } \\
2010\end{array}$ & - & 6 & 0 & 5 & 6 & 100 \\
\hline & $\begin{array}{c}\text { Venezuela III, } \\
2007\end{array}$ & $\begin{array}{l}\text { Caracas } \\
(2007)\end{array}$ & 2 & 0 & 4 & 12 & 53,33 \\
\hline \multirow{2}{*}{$\begin{array}{c}\text { Dificultad media } \\
(20,43-50,45)\end{array}$} & $\begin{array}{c}\text { Ecuador III, } \\
2010\end{array}$ & $\begin{array}{l}\text { Quito } \\
\text { (2010) }\end{array}$ & 5 & 2 & 2 & 8 & 45,56 \\
\hline & $\begin{array}{c}\text { Venezuela II, } \\
2003\end{array}$ & - & 2 & 0 & 4 & 10 & 44,44 \\
\hline \multirow{9}{*}{$\begin{array}{l}\text { Dificultad baja } \\
\text { (20,42 a menos) }\end{array}$} & $\begin{array}{c}\text { Colombia II, } \\
2002\end{array}$ & $\begin{array}{l}\text { Bogotá } \\
(2013)\end{array}$ & 1 & 1 & 6 & 4 & 13,89 \\
\hline & $\begin{array}{c}\text { Ciudad de } \\
\text { Buenos Aires, } \\
2000\end{array}$ & $\begin{array}{l}\text { Buenos } \\
\text { Aires } \\
(2005, \\
2014)\end{array}$ & 3 & 1 & 2 & 2 & 7,22 \\
\hline & $\begin{array}{c}\text { Ecuador I, } \\
1998\end{array}$ & - & 5 & 1 & 5 & 0 & 0,55 \\
\hline & $\begin{array}{c}\text { Colombia I, } \\
1994\end{array}$ & $\begin{array}{l}\text { Bogotá } \\
(1999)\end{array}$ & 1 & 1 & 6 & 0 & 0,55 \\
\hline & $\begin{array}{l}\text { Bolivia I, } \\
2009\end{array}$ & - & 6 & 0 & 3 & 0 & 0 \\
\hline & $\begin{array}{l}\text { Ecuador II, } \\
\quad 2008\end{array}$ & - & 5 & 0 & 2 & 0 & 0 \\
\hline & $\begin{array}{l}\text { Ciudad de } \\
\text { Córdoba, } \\
2002\end{array}$ & $\begin{array}{c}\text { Córdoba } \\
\text { (2002) }\end{array}$ & 4 & 0 & 2 & 0 & 0 \\
\hline & $\begin{array}{c}\text { Venezuela I, } \\
1999\end{array}$ & - & 2 & 0 & 4 & 0 & 0 \\
\hline & Perú, 1994 & $\begin{array}{l}\text { Lima } \\
(2013)\end{array}$ & 5 & 0 & 1 & 0 & 0 \\
\hline \multicolumn{3}{|c|}{ Promedio } & 3,62 & 0,46 & 3,54 & 3,23 & 20,43 \\
\hline
\end{tabular}

Fuente: Elaboración propia sobre la base de revisión normativa y bibliográfica. 


\section{b. El rol de los actores políticos, apoyo a la autoridad y causas para la activación del mecanismo de revocatoria}

La mayoría de iniciativas de revocatoria fueron promovidas desde arriba (top down), por la autoridad cuestionada y, principalmente, por opositores a la gestión edil (véase Cuadro 7). En el caso de Buenos Aires (2005), la autoridad —el jefe de gobierno, Aníbal Ibarra — impulsó la recolección de firmas, con la finalidad de que la ciudadanía expresara su aprobación o rechazo hacia la gestión en la capital, tras un accidente en una discoteca del barrio de Once (Bottinelli, 2005). Sin embargo, no se alcanzó el porcentaje de firmas necesario, de 500 mil aproximadamente (Arques, 2014a). Por su parte, tanto en Bogotá (2000 y 2013) como en Lima (2013), las revocatorias fueron promovidas por excandidatos y opositores políticos.

Cuadro 7. Origen de promoción de la revocatoria

\begin{tabular}{|c|c|c|c|c|c|}
\hline Origen & $\begin{array}{l}\text { Actor } \\
\text { promotor }\end{array}$ & $\begin{array}{c}\mathrm{N}^{\circ} \\
\text { iniciativas }\end{array}$ & Casos & Causas & $\begin{array}{c}\text { Actor que solicitó } \\
\text { revocatoria }\end{array}$ \\
\hline \multirow{4}{*}{$\begin{array}{l}\text { Desde } \\
\text { arriba }\end{array}$} & $\begin{array}{c}\text { Autoridad } \\
\text { cuestionada }\end{array}$ & 1 & $\begin{array}{c}\text { Buenos } \\
\text { Aires (2005) }\end{array}$ & $\begin{array}{c}\text { Manifestación de la apro- } \\
\text { bación o rechazo hacia la } \\
\text { gestión }\end{array}$ & $\begin{array}{l}\text { Jefe de gobierno } \\
\text { Aníbal Ibarra }\end{array}$ \\
\hline & \multirow{3}{*}{$\begin{array}{l}\text { Opositores } \\
\text { políticos }\end{array}$} & \multirow{3}{*}{3} & $\begin{array}{l}\text { Bogotá } \\
(1999)\end{array}$ & $\begin{array}{l}\text { Rechazo a algunas obras } \\
\text { específicas }\end{array}$ & $\begin{array}{l}\text { Excandidatos y opo- } \\
\text { sitores políticos }\end{array}$ \\
\hline & & & $\begin{array}{l}\text { Bogotá } \\
(2013)\end{array}$ & $\begin{array}{c}\text { Ineficiencia de la gestión } \\
\text { y rechazo a algunas obras } \\
\text { específicas }\end{array}$ & $\begin{array}{c}\text { Congresista del } \\
\text { Partido de gobierno y } \\
\text { opositores políticos }\end{array}$ \\
\hline & & & Lima (2013) & Ineficiencia de la gestión & $\begin{array}{l}\text { Partido político del } \\
\text { exalcalde de Lima }\end{array}$ \\
\hline \multirow{2}{*}{$\begin{array}{l}\text { Desde } \\
\text { arriba }\end{array}$} & \multirow{2}{*}{ Ciudadanía } & \multirow{2}{*}{2} & $\begin{array}{l}\text { Córdoba } \\
\text { (2002) }\end{array}$ & $\begin{array}{l}\text { Cuestionamientos a la } \\
\text { gestión }\end{array}$ & Un ciudadano \\
\hline & & & $\begin{array}{l}\text { Quito } \\
(2010)\end{array}$ & $\begin{array}{l}\text { Incumplimiento de pro- } \\
\text { mesas de campaña }\end{array}$ & $\begin{array}{l}\text { Dirigente de barrios } \\
\text { marginales de Quito* }\end{array}$ \\
\hline \multirow{2}{*}{$\begin{array}{l}\text { Desde } \\
\text { abajo }\end{array}$} & $\begin{array}{c}\text { Ciudadanía/ } \\
\text { opositores } \\
\text { políticos }\end{array}$ & 1 & $\begin{array}{c}\text { Buenos } \\
\text { Aires (2014) }\end{array}$ & $\begin{array}{l}\text { Rechazo a algunas obras } \\
\text { específicas }\end{array}$ & $\begin{array}{l}\text { Cinco vecinos de } \\
\text { Buenos Aires* }\end{array}$ \\
\hline & $\begin{array}{c}\text { No se } \\
\text { especifica }\end{array}$ & 1 & $\begin{array}{l}\text { Caracas } \\
(2007)\end{array}$ & - & - \\
\hline
\end{tabular}

Fuente: Elaboración propia sobre la base de revisión bibliográfica, de datos oficiales en instituciones electorales y en medios de prensa escrita en versión digital.

* Información extraída de medios de prensa virtuales. 
En 1999 se promovió la revocatoria del alcalde de Bogotá Enrique Peñalosa (1997-2000), del Movimiento Cívico Por la Bogotá que Soñamos. Peñalosa sufría de un notable descrédito al final de su primer año (Bromberg, 2013), con una impopularidad de 85\% en marzo de 1999 (Diario Semana, 2000); por lo cual distintos activistas, entre los cuales se encontraban los vendedores ambulantes, se mostraron interesados en apoyar la recolección de firmas para activar la consulta en oposición a los desalojos para recuperar el espacio público y para realizar obras. La solicitud de revocatoria fue promovida por el excandidato a la alcaldía de Bogotá del Partido Liberal, Luis Fernando Rosas (Redacción El Tiempo, 1999b); el representante de la Cámara por Bogotá por el Movimiento Reconstrucción Democrática Nacional (1998-2002), Germán Navas Talero; el concejal de la capital por el Polo Democrático, Bruno Díaz (Lancheros, 2012), entre otros (Franco-Cuervo, 2014a, pp. 67-68).

En 2009 se promovió un intento de revocatoria en contra del alcalde de Bogotá, Samuel Moreno (2008-2011), del Polo Democrático Alternativo, debido a la insatisfacción ciudadana frente a la mala administración de la ciudad. No obstante, esta no llegó a presentarse ante la Registraduría por falta de recursos y escasas condiciones de seguridad, dado que fue impulsada por un movimiento exclusivamente ciudadano (Uribe, 2016). Posteriormente, en 2013, se presentó un intento de revocatoria formal en contra del alcalde Gustavo Petro (2012-2015) del Movimiento Progresistas. Esta iniciativa fue impulsada en un contexto de vertiginoso descenso de su popularidad, pues pasó del 74\% de aprobación en 2012, al 41\% en enero de 2013, debido al cambio de modelo de recolección de basuras de la ciudad (Uribe, 2016, p. 189). La iniciativa de revocatoria fue promovida por Miguel Gómez Martínez, representante del Partido de la U (Lancheros, 2012), agrupación política que quedó en segundo lugar en las elecciones de 2011 en alianza con el Partido Verde; aduciendo ineficiencia en la gestión, así como deterioro del transporte en la ciudad y en el sistema de recolección de basura, entre otros (Rey, 2015, p. 11). Gómez promovió la revocatoria sin el apoyo de su partido, en coordinación con el exalcalde de la capital (1992-1994) y candidato a la alcaldía de Bogotá en las elecciones de 2011 Jaime Castro (Partido Liberal), como venganza por la revocatoria promovida en su contra por Petro en 1994. Castro se convirtió en uno de los actores claves de la iniciativa de revocatoria, ya que desde noviembre de 2013 coordinó con Gómez para promover la revocatoria y se encargó de promover reuniones, foros, programas y columnas a favor de la revocatoria; mientras que Gómez se abocó a la recolección de firmas (Ardila Arrieta, 2013). Del mismo modo, la campaña a favor del Sí fue liderada por el exvicepresidente 
durante los gobiernos de Álvaro Uribe, Francisco Santos (2002-2010) (El País, $11 / 02 / 2014)$, y apoyada por siete iniciativas ciudadanas, que configuraron la plataforma «Bogotá no se rinde»"

Tras superar la verificación y la validación del porcentaje de firmas necesario por la RNEC, con lo cual se activó la revocatoria de Petro, el alcalde y sus seguidores iniciaron procesos judiciales para evitar la consulta ciudadana. Empero, un hito que marcó el proceso fue el hecho de que la Procuraduría General de la Nación destituyó e inhabilitó a Petro para ejercer cargo públicos por quince años, debido al cambio de modelo de recolección de basuras en la capital colombiana. Este fallo fue apelado, ante lo cual la Procuraduría falló en segunda instancia la destitución e inhabilitación del alcalde, con lo cual se canceló el proceso de revocatoria a principios de enero de 2013. No obstante, «el 23 de enero, un falló de tutela ante el Tribunal Administrativo de Cundinamarca suspendió el proceso de destitución e inhabilidad que cursaba en contra del burgomaestre y, por lo tanto, la revocatoria se volvió a activar» (Uribe, 2016, p. 196). En marzo de 2014, el Consejo de Estado - que constituye la máxima autoridad administrativa en Colombia— volvió a destituir e inhabilitar a Petro; ante lo cual «la Comisión Interamericana de Derechos Humanos expidió medidas cautelares a favor del alcalde» (Uribe, 2016, p. 191). Así pues, en mayo de 2015 se anuló definitivamente el proceso de revocatoria, por solicitud de la RNEC, argumentando que no se justificaba gastar 338 mil millones de pesos en una consulta de revocatoria, dado que el periodo de mandato de la autoridad estaba a punto de culminar.

De otro lado, en 2013 se solicitó la revocatoria de la alcaldesa de Lima Susana Villarán (2011-2014), de Fuerza Social, y la de todos los integrantes del Concejo Municipal de Lima Metropolitana, alegando ineficiencia en el desempeño de las funciones (ONPE, 2013c, p. 40). Esta solicitud se presentó en un contexto de creciente desaprobación ciudadana hacia la gestión edil, que ascendió al 70\% en noviembre de 2012 (Ipsos Apoyo 2012, p. 5). Los promotores de la consulta fueron Carlos Vidal y Marco Tulio Gutiérrez, este último fue regidor de Lima por Izquierda Unida (1980-1983) y estaba vinculado al partido Solidaridad Nacional, agrupación política del alcalde de Lima Luis Castañeda — quien asumió la alcaldía de Lima por dos periodos conse-

\footnotetext{
9 Entre estos grupos ciudadanos figuran «Bogotá somos todos», «Fuera Petro», «Dónde le firmo», "Creo Colombia», «Revoquemos al Alcalde Petro», «No más Petro», «Movimiento Ciudadano» y «Movimiento firme con Bogotá», este último liderado por Miguel Gómez. Además, se buscó el apoyo de Uldarico Peña, que dirigía cerca de $40 \%$ de taxis en la capital, y de Éverth Bustamante, exmiembro del M-19; aunque finalmente solo se adhirió la segunda (Ardila Arrieta, 2013).
} 
cutivos, de 2003 a 2010 y, posteriormente, fue reelegido en 2014-, puesto que había prestado servicios a la alcaldía durante su gestión (Vásquez Oruna, 2014a, p. 45). De manera encubierta, dicha agrupación participó en la campaña a favor del Sí (Redacción La República, 2013). Asimismo, la campaña fue apoyada por distintos partidos políticos, entre los cuales figuran Unión por el Perú y el Partido Aprista Peruano, al igual que algunos congresistas de Fuerza Popular (fujimorismo), como Julio Gagó y Martha Moyano, pues la dirigencia del partido declaró que dejaba a discreción de los militantes si apoyaban la revocatoria (Cavero, 2013, p. 112). A su vez, los transportistas, comerciantes ambulantes del centro de Lima, pequeños empresarios de imprentas, entre otros sectores sociales, también se mostraron interesados en promover la consulta (Vásquez Oruna, 2014a, p. 45).

En la línea anterior, se observa que, si bien en algunos casos existía insatisfacción popular hacia las gestiones municipales, fueron minoritarios los intentos de revocatoria promovidos desde abajo (bottom up) en las ciudades grandes de América del Sur. En efecto, únicamente se registraron dos intentos de revocatoria impulsados por ciudadanos o miembros de alguna asociación ciudadana, donde la ciudadanía habría jugado un rol protagónico en la activación de este MDD. El primero fue en la ciudad de Córdoba (2002), en Argentina, donde un ciudadano solicitó la revocatoria del intendente Germán Kammerath, debido a serios cuestionamientos hacia su gestión y solicitudes de juicio político por presunta administración fraudulenta (Clarín, 2003). Sin embargo, la autoridad cuestionada apeló ante el Tribunal Electoral provincial, el cual sentenció que el juicio político en contra del intendente anulaba el pedido de revocatoria (Welp, 2014; Arques, 2014a). El segundo caso tuvo lugar en Quito (2010), donde el dirigente de barrios marginales —el vicepresidente coordinador de barrios de Chillogallo- Nemo Domínguez, solicitó la revocatoria de mandato del alcalde Augusto Barrera por incumplimiento de ofertas de campaña (La Hora, 2010); empero, el CNE no admitió recolección de firmas debido a la imprecisión en la formación y precisión del motivo por el cual se pretendía realizar la revocatoria.

Por su parte, la solicitud de revocatoria de 2014, en Buenos Aires, representa un caso especial. Si bien el proceso de revocatoria en contra del jefe de gobierno Mauricio Macri y de la vicejefa María Eugenia Vidal fue promovido por cinco ciudadanos, algunos de ellos pertenecían a una agrupación política que compitió en las elecciones de 2011, donde resultaron perdedores. Se presentaron los intentos de revocatoria aduciendo fallas en la gestión: represión en el Hospital Borda, falta de obras para mitigar las inundaciones y el dictado de decreto de 
necesidad y urgencia que aprobó el régimen de defensa de la libertad de expresión en la capital; todas las cuales fueron juntadas en un mismo expediente por el Tribunal Supremo de Justicia (TSJ) de Buenos Aires. Sin embargo, dos de las cinco personas que presentaron las iniciativas de revocatoria — Lucía Medina y Ricardo Pelusa - eran militantes del partido político Aluvión Ciudadano (Infobae, 2014).

Así pues, únicamente dos casos de los que registraron intentos de revocatoria en ciudades grandes de Sudamérica fueron promovidos por la ciudadanía, empleándose el mecanismo de revocatoria de mandato como una herramienta de accountability vertical en manos de los representados. Empero, cinco casos evidencian el uso - ya sea de forma abierta o encubierta - por parte de los excandidatos y opositores políticos tanto locales como nacionales, en busca de desprestigiar a sus contrincantes y ganar espacios políticos en ciudades grandes que, por lo general, han sido las ciudades capitales de estos países. Más aún, únicamente los intentos de revocatoria en Lima (2013) y Bogotá (2013) lograron cumplir con los requisitos establecidos por ley. Sin embargo, tan solo en el primer caso se hizo efectiva la consulta popular; por tanto, a continuación se abordarán los factores que influyeron en la efectividad del mecanismo de la revocatoria en Lima.

\section{c. Lima (2013) en perspectiva comparada}

Cabe destacar las particularidades de la revocatoria en Lima Metropolitana, porque se buscaba destituir a la alcaldesa Susana Villarán, pero se aprobó la consulta para otros 39 funcionarios (Del Águila, 2013, p. 6); aunque tan solo la alcaldesa y los regidores de oposición — con la excepción del hijo del alcalde Castañeda Lossio- se mantuvieron en sus cargos. Este aspecto se vincula con el diseño institucional de la revocatoria vigente en ese momento, debido a la gran facilidad para su activación.

El nivel de dificultad para activar la revocatoria aplicado en Lima era similar al de Córdoba, donde la iniciativa fue promovida por un ciudadano, con lo cual se podría haber resuelto una crisis ante una gestión edil muy cuestionada y envuelta en pedidos de juicio político (Arques, 2014a, p. 175); aunque finalmente se interpusieron trabas legales (Welp, 2014, p. 38). Del mismo modo, el nivel de dificultad del diseńo institucional aplicado en la capital peruana fue similar al que rigió los procesos en Buenos Aires $(2005,2014)$ y, parcialmente, Bogotá (2013), aunque en estos casos se promovió la revocatoria desde arriba, por la propia autoridad cuestionada y por actores vinculados a partidos políticos, respectivamente. 
En esta línea, la revocatoria en Lima da cuenta de que en ciudades grandes de América de Sur — caracterizadas por concentrar alrededor del 30\% de la población nacional—, por más que el diseño institucional sea menos exigente para su activación desde la ciudadanía (bottom-up), existen escasos incentivos para que una organización de la sociedad civil la promueva debido a la limitada capacidad de organización con la que suelen contar estas agrupaciones. La recolección de 400 mil firmas representa un gran reto que difícilmente podría ser afrontado por un grupo de ciudadanos organizados, tomando en cuenta la gran debilidad y fragmentación de la sociedad civil desde la década de 1990 (Remy, 2005). De manera similar al caso colombiano, donde la sociedad civil se encuentra poco organizada, de tal forma que les permita exigir una genuina rendición de cuentas y realizar un control político efectivo (Franco-Cuervo, 2014, pp. 72-73). Ello evidencia que resulta fundamental que la iniciativa de revocatoria sea promovida por una organización política con capacidad suficiente de movilizar los recursos tanto económicos como logísticos para desplegar el proceso de recolección de firmas necesarias según la normativa.

Sin embargo, a diferencia de los demás países de la subregión, e incluso del caso colombiano que registra altos niveles de competencia política, la particularidad del diseño peruano recientemente reformado (2015) radica en que se establecía la necesidad de realizar nuevas elecciones municipales en caso de revocar a más de un tercio de los miembros del concejo municipal. Esta característica se convirtió en un incentivo fundamental para que el origen de la revocatoria se dé desde los partidos políticos en busca de (re)tomar el control de la gestión edil, de tal forma que este MDD no habría permitido distribuir el poder en las democracias representativas, sino que más bien los opositores políticos utilizaron este mecanismo como una estrategia para movilizar electores en potencia (Welp y Serdült, 2012), con la finalidad de retomar el poder en la alcaldía de Lima — en el caso de Solidaridad Nacional — y de adquirir visibilidad política a nivel nacional, en el caso de los partidos políticos de oposición.

Más aún, si bien el diseño institucional del mecanismo de revocatoria de mandato y el origen de la iniciativa influyen en la efectividad del mismo; estas constituyen condiciones necesarias, mas no suficientes. En efecto, al igual que en Córdoba (2002) y Bogotá (1999 y 2013), la iniciativa de revocatoria en contra de Susana Villarán se incorporó en la agenda política en un contexto de creciente insatisfacción ciudadana (Welp, 2015, p. 14), como consecuencia del acelerado descenso de la aprobación de la gestión. En octubre del mismo año, la desaprobación ascendió al 77\% (véase Gráfico 1); lo cual contrasta significativamente con el nivel de aprobación de la gestión del alcalde anterior, Luis 
Castañeda (Solidaridad Nacional), cuyo punto más alto de desaprobación fue del 32\%, hacia mediados de su último año en la alcaldía (Ipsos, 2010).

Gráfico 1. Aprobación de la gestión de Susana Villarán, 2011-2013

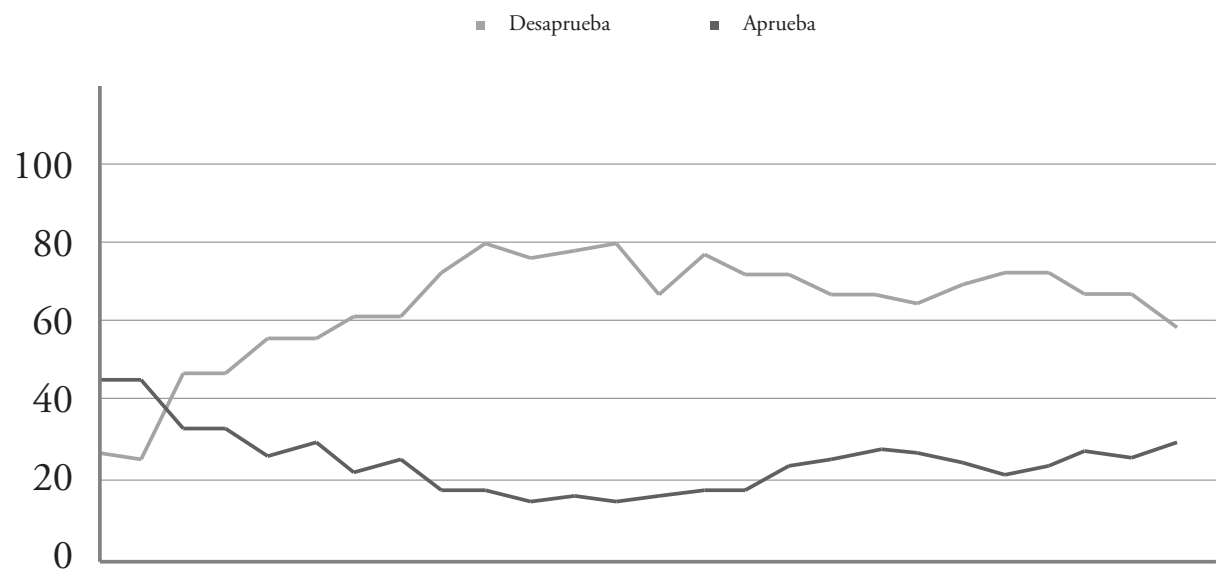

Ene11 Abr11 Iul11 Oct11 Ene12 Abr12 Iul12 Oct12 Ene13

Fuente: JNE-Infogob. Elaboración propia.

El caso de Lima evidencia el gran impacto del nivel de desaprobación ciudadanos hacia la gestión municipal en la efectividad del mecanismo de revocatoria, puesto que — por más que la iniciativa haya estado motivada por intereses particulares - este factor permitió que los promotores de la revocatoria logren capitalizar los elevados niveles de insatisfacción social. De esta forma, no solo se encuentran en capacidad de recabar las firmas necesarias para activar la revocatoria de mandato, sino también de sumar apoyos desde distintas organizaciones de la sociedad civil y grupos de ciudadanos.

En la línea anterior, resulta fundamental tomar en cuenta la capacidad para llevar a cabo la acción colectiva, tanto desde la ciudadanía como desde las agrupaciones políticas opositoras frente a las autoridades cuestionadas. Al respecto, también sería importante tomar en cuenta, por un lado, la neutralidad de los organismos electorales encargados de activar el proceso y realizar la consulta, puesto que los casos de Venezuela y Colombia evidencian ciertas irregularidades en el proceso - ya sea para facilitar o dificultar la activación de dicho MDD — y, de otro lado, el impacto de los medios de comunicación — tanto convencionales como digitales - en las campañas a favor o en contra de la revocatoria. 


\section{Conclusiones}

En aquellos países de América del Sur que han incluido el mecanismo de revocatoria en su normativa, es limitada la cantidad de iniciativas por activar este MDD en ciudades grandes. Tan solo en ocho ciudades con más de 1 millón de habitantes se han presentado solicitudes de revocatoria y, en su mayoría, estas se han dado en las ciudades capitales. Más aún, por lo general, las solicitudes de revocatoria se vieron frustradas al no cumplir con la presentación de la cantidad de firmas necesarias, ya que este suele ser el requisito más exigente para activar este mecanismo.

Asimismo, a partir de un análisis en perspectiva comparada del caso de revocatoria en Lima (2013), el presente estudio demuestra que la participación de la ciudadanía a través de la activación del mecanismo de revocatoria en ciudades grandes de América del Sur no suele darse de manera directa; razón por la cual difícilmente se podría hablar de un MDD que permita que los ciudadanos se conviertan en tomadores de decisiones, ni de que esta institución participativa contribuya con una distribución del poder en las democracias representativas. Si bien la ciudadanía cuenta con la potestad de decidir directamente a través del sufragio universal y secreto respecto a la continuidad de alguna autoridad elegida popularmente, la efectividad de la aplicación de este mecanismo no depende exclusivamente de la participación desde abajo (bottom-up).

Por el contrario, a pesar de que, en términos generales, es bajo el nivel de dificultad para la activación de la revocatoria en la mayoría de países de Sudamérica; los ciudadanos cuentan con escasos incentivos para promover la utilización de este mecanismo, debido a los costos que supone el cumplir con los requisitos para activar dicho MDD en comparación con las recompensas que supone su utilización. Por lo tanto, en la mayoría de los casos, la población se encuentra sujeta a la solicitud de revocatoria iniciada por los partidos políticos o por políticos particulares a nivel local, cuya utilización representa una estrategia de los partidos para movilizar electores para futuras contiendas electorales, configurándose, como señala Del Águila (2013), en una herramienta de «perpetuación electoral». En esta línea, esta institución de participación no cambia la democracia representativa, como sugieren Cameron et al. (2012), puesto que se mantiene e incluso se puede llegar a acentuar el poder de los actores políticos tradicionales, como los partidos políticos, en un contexto de creciente insatisfacción hacia la política y las autoridades locales.

En esta línea, si bien, como señala Welp (2015), la posibilidad de éxito de la revocatoria puede explicarse por la combinación del diseńo institucional del 
proceso y el nivel de institucionalización del sistema de partidos; el presente estudio da cuenta de que el segundo factor tiene un mayor impacto en la efectividad de la aplicación de este mecanismo en ciudades grandes de América del Sur. En específico, a diferencia de las circunscripciones pequeñas donde es más común el uso de la revocatoria de mandato, facilitada por una reducida población electoral; en las ciudades grandes se observa de manera más clara la importancia de la interacción entre los actores sociales y políticos, así como los recursos financieros y logísticos con los que cuentan los distintos grupos, en contextos de elevada competencia y fragmentación política, y de creciente insatisfacción ciudadana respecto a la gestión y a las autoridades a nivel local.

Así pues, la efectividad de la revocatoria en ciudades grandes de América del Sur dependería de una conjunción, principalmente, de dos condiciones necesarias. En primer lugar, resulta fundamental contar con elevados niveles de insatisfacción ciudadana hacia la gestión local para que la revocatoria de mandato se haga efectiva, ya que los ciudadanos estarán interesados en manifestar su disconformidad hacia la autoridad, sin la necesidad de esperar nuevas elecciones para castigar o premiar al mandatario y/o a su partido. En segundo lugar, la promoción de la iniciativa de revocatoria requiere ser liderada por una organización política con capacidad suficiente para cumplir con los requisitos para su activación, ya que una estructura ciudadana difícilmente lo podría lograr debido a la cantidad de electores de las ciudades grandes, así como el nivel de complejidad del escenario político y social.

\section{BibLIOGRAFÍA}

Altman, D. (2010). Plebiscitos, referendos e iniciativas populares en América Latina: ¿mecanismos de control político o políticamente controlados? Perfiles Latinoamericanos, 18(35), 9-34.

Altman, D. (2011). Direct democracy worldwide. Nueva York, NY : Cambridge University Press. doi: $10.1017 /$ cbo9780511933950

Ardilla Arrieta, L. (2013). La revocatoria a Petro por dentro. La Silla Vacía. Recuperado de http:// lasillavacia.com/node/43852

Arques, F. (2014a). La revocatoria de mandato a nivel subnacional en Argentina. En F. Tuesta Soldevilla (Ed.), Una onda expansiva. Las revocatorias en el Perú y América Latina (pp. 157-186). Lima, Perú: Jurado Nacional de Elecciones, Pontificia Universidad Católica del Perú.

Arques, F. (2014b). Argentina: una herramienta de los gobernados en manos de los gobernantes. En Y. Welp y U. Serdült (coords.), La dosis hace el veneno. Análisis de la revocatoria del mandato en América Latina, Estados Unidos y Suiza (pp. 159-186). Quito, Ecuador: Consejo Nacional Electoral República Ecuador, Instituto de la Democracia.

Battle, M. (2014). Competencia multinivel en el sistema de partidos colombiano: Del bipartidismo tradicional al predominio de nuevos actores (1992-2011). En F. Freidenbergy J. Suárez-Cao 
(Eds.), Territorio y poder. Nuevos actores y competencia politica en los sistemas de partidos multinivel en América Latina (pp. 93-123). Salamanca, España: Ediciones Universidad de Salamanca.

Benton, A. L. (2012). Bottom-Up Challenges to National Democracy. Mexico's (Legal) Subnational Authoritarian Enclaves. Comparative Politics, 44(3), 253-271. doi: $10.5129 / 001041512800078931$

Breuer, A. (2007). Institutions of Direct Democracy and Accountability in Latin America's Presidential Democracies. Democratization, 14(4), 554-579. doi: 10.1080/13510340701398287

Bromberg, P. (2013). La revocatoria del mandato de Petro: hagan sus apuestas. Razonpublica. com. Recuperado de http://www.razonpublica.com/index.php/regiones-temas31/3492-la-revocatoria-del-mandato-de-petro-hagan-sus-apuestas.

Bustos, C. (2002). Mecanismos de participación democrática: Ficción o realidad. Revista Facultad de Ciencias Económicas: Investigación y Reflexión, 1, 68-77.

Cadena, A., Remes, J., Manyika, J., Dobbs, R., Roxburgh, C., Elstrodt, H-P., Chaia, A., Restrepo, A. (2011). Construyendo ciudades competitivas. La clave para el crecimiento en América Latina. Washington, DC: McKinsey Global Institute.

Cameron, M., Hershberg, E. y Sharpe, E. K. (2012). New Institutions for Participatory Democracy in Latin America. Voice and Consequence. Nueva York, NY: Palgrave Macmillan. doi: $10.1057 / 9781137270580$

Castellanos Santamaría, A. S. (2014). Ecuador: la transformación de las reglas del juego y sus consecuencias (1998-2013). En Y. Welp y U. Serdült (coords.), La dosis hace el veneno. Análisis de la revocatoria del mandato en América Latina, Estados Unidos y Suiza (pp. 83-110). Quito, Ecuador: Consejo Nacional Electoral de la República del Ecuador, Instituto de la Democracia.

Cavero Cornejo, O. (2013). ¿Cómo explicar la campaña para revocar a la alcaldesa de Lima Metropolitana, Susana Villarán? Una hipótesis sobre el poder y la política en la capital. Debates en Sociología, 38, 109-128.

Dalton, R. J., Burklin, W. P. y Drummond, A. (2001). Public Opinion and Direct Democracy. Journal of Democracy, 12(4), 141-153. doi: 10.1353/jod.2001.0066

Del Águila, A. (2013). La revocatoria en análisis comparado: notas para la reforma en el Perú. Revista Argumentos, 2, 3-8. Recuperado de http://revistaargumentos.iep.org.pe/articulos/ la-revocatoria-en-analisis-comparado-notas-para-la-reforma-en-el-peru/

Eberhardt, M. L. (2013). Crisis de representación en las democracias presidencialistas latinoamericanas. ¿La revocatoria del mandato como opción? Elecciones, 12(13), 15-51.

Franco-Cuervo, A. B. (2014a). Colombia: instituciones, líderes políticos y abstención electoral (1991-2013). En Y. Welpy U. Serdült (coords.), La dosis hace el veneno. Análisis de la revocatoria del mandato en América Latina, Estados Unidos y Suiza (pp. 57-81). Quito, Ecuador: Consejo Nacional Electoral de la República del Ecuador, Instituto de la Democracia.

Franco-Cuervo, A. B. (2014b). La revocatoria en Colombia: a propósito de la iniciativa de revocatoria del alcalde mayor de Bogotá. En F. Tuesta Soldevilla (ed.), Una onda expansiva. Las revocatorias en el Perú y América Latina (pp. 99-134). Lima, Perú: JNE, PUCP.

Guzmán Hernández, T. Y. (2014). Cuba: deudas pasadas y retos presentes desde la norma. En Y. Welpy U. Serdült (coords.), La dosis hace el veneno. Análisis de la revocatoria del mandato en América Latina, Estados Unidos y Suiza (pp. 187-205). Quito, Ecuador: Consejo Nacional Electoral República Ecuador, Instituto de la Democracia.

Jiménez, W. (2001). Revocatoria del mandato: experiencias, dificultades y ajustes necesarios. Territorios, 5, 35-48.

Kaufmann, B. y Waters, D. M. (2004). Direct Democracy in Europe. Durham, NC, United States: Carolina Academic Press. 
Kaufmann, B., Büchi, R. y Bran, N. (2008). Guidebook to Direct Democracy in Switzerland and Beyond. 2008 Edition. Berna, Suiza: Benteli Hallwag Druck AG.

Kornblith, M. (2009). La revocatoria de mandato: Lecciones a partir de la experiencia venezolana. (Working paper $\mathrm{N}^{\circ} 358$ ). The Helen Kellogg Institute for International Studies.

Kornblith, M. (marzo de 2007). Democracia Directa y Revocatoria de Mandato en Venezuela. En Presidencia. Conferencia Internacional Democracia Directa en América Latina. Center for Research on Direct Demoracy (C2D), la Universidad de San Martín (UNSAM) y el Instituto para la Democracia y la Asistencia Electoral (IDEA Internacional). Conferencia llevada a cabo en Buenos Aires, Argentina.

Kornblith, M. (2014a). Revocatoria del mandato presidencial en Venezuela: Definición y puesta en práctica. En A. Lissidini, Y. Welp y D. Zovatto (comps.), Democracias en movimiento. Mecanismos de democracia directa y participativa en América Latina (pp.131-166). México D.F, México: UNAM, Centro de Investigaciones sobre democracia directa, Instituto para la democracia y la asistencia electoral.

Kornblith, M. (2014b). Revocatoria del mandato en Venezuela. Examen de los procesos presidencial, regional y local. En F. Tuesta (ed.), Una onda expansiva. Las revocatorias en el Perú y América Latina (pp. 209-248). Lima, Perú: JNE, PUCP.

Lissidini, A. (2007). ¿Cómo investigar la democracia directa? Explicaciones, interpretaciones y prejuicios. (Documento de Trabajo No 27). Escuela de Política y Gobierno, UNSAM.

Martínez Cárdenas, E. E. (2013). Revocatoria de mandato: ¿ataque a la democracia representativa?. Reflexión Política, 15(29), 84-96.

MOE (Misión de Observación Electoral) (2012). Mecanismos de participación ciudadana en Colombia. 20 años de ilusiones. Bogotá, Colombia: Torre Blanca Agencia Gráfica.

Moncada, E. (2011). Subnational Comparative Research on Democracy: Taking Stock and Looking Forward. APSA-CD: The Newsletter of the Comparative Democratization Section of the American Political Science Association (APSA).

ONPE (2013a). Nuevas Elecciones municipales y Segunda Consulta Popular de Revocatoria del Mandato de Autoridades Municipales julio 2013. Lima, Perú: ONPE.

ONPE (2013b). Consulta popular de revocatoria en el ámbito provincial. Hacia una comprensión de los escenarios y actores (2004, 2008 y 2012). Lima, Perú: ONPE.

ONPE (2013c). Consulta Popular de Revocatoria del Mandato de Autoridades Municipales de marzo 2013. Documento de gestión. Lima, Perú: ONPE.

Papadopolus, Y. (1995). Analysis of Functions and Dysfunctions of Direct Democracy: Top-Down and Bottom-Up Perspectives. Politics \& Society, 23(4), 421-448.

Qvortrup, M. (2014). La experiencia estadounidense de 19776 a 2012. En Y. Welp y U. Serdült (coords.), La dosis hace el veneno. Análisis de la revocatoria del mandato en América Latina, Estados Unidos y Suiza (pp. 207-224). Quito, Ecuador: Consejo Nacional Electoral de la República de Ecuador, Instituto de la Democracia.

Ramírez, F. (2014). Del vértigo al estancamiento: los procesos revocatorios locales en Ecuador. En F. Tuesta Soldevilla (ed.), Una onda expansiva. Las revocatorias en el Perú y América Latina (pp. 135-156). Lima, Perú: Jurado Nacional de Elecciones, Pontificia Universidad Católica del Perú.

Ramírez, F. y Welp, Y. (2011). Presentación del dossier: Nuevas instituciones participativas y democráticas en América Latina. Íconos. Revista de Ciencias Sociales, (40), 11-20.

Remy, M. I. (2005). Los múltiples campos de la participación ciudadana en el Perú: un reconocimiento del terreno y algunas reflexiones. Lima. Perú: Instituto de Estudios Peruanos.

Remy, M. I. (2008). Y se vienen las revocatorias. Revista Argumentos, 2(1), S/P Recuperado de http://www.revistargumentos.org.pe/y_se_vienen_las_revocatorias.html 
Remy, M. I. (2013). La revocatorias en el Perú: entre la participación masiva y la debilidad institucional. Revista Argumentos, 7(1), 29-37. Recuperado de http://www.revistargumentos.org.pe/ revocatorias.html

Rey, J. (2015). Entre la revocatoria y la destitución. Análisis del frustrado intento de revocar al alcalde de Bogotá. (C2D Working Paper Series). Centre for Research on Direct Democracy. University of Zurich.

Rivera, J. L. (2006). Revocatoria del mandato para funcionarios de elección popular en los gobiernos locales. Revista de Derecho Electoral, 2, 1-42.

Scarrow, S. E. (2001). Direct Democracy and Institutional Change: A Comparative Investigation, Comparative Political Studies, 34(6), 651-665.

Schneider, C. y Welp, Y. (2015). Diseños institucionales y (des)equilibrios de poder: las instituciones de participación ciudadana en disputa. Revista Mexicana de Ciencias Politicas y Sociales, 60(224), 15-44. doi:10.1016/S0185-1918(15)30002-7

Serdült, U. (2014). Una institución durmiente: historia, normas legales y prácticas de la revocatoria en Suiza. En Y. Welp y U. Serdült (coords.), La dosis hace el veneno. Análisis de la revocatoria del mandato en América Latina, Estados Unidos y Suiza (pp. 225-246). Quito, Ecuador: Consejo Nacional Electoral República Ecuador, Instituto de la Democracia.

Serdült, U. y Welp, Y. (2012). Direct Democracy Upside Down. Taiwan Journal of Democracy, 8(1), 69-92.

Smulovitz, C. (2001). Judicialización y accountability xocial en Argentina. Recuperado de http:// lasa.international.pitt.edu/Lasa2001/SmulovitzCatalina.pdf

Tuesta, F. (2014a). Perú: entre la participación y la gobernabilidad local (1997-2013). En Y. Welp y U. Serdült (coords.), La dosis hace el veneno. Análisis de la revocatoria del mandato en América Latina, Estados Unidos y Suiza (pp. 7-30). Quito, Ecuador: Consejo Nacional Electoral de la República del Ecuador, Instituto de la Democracia.

Tuesta, F. (2014b). La revocatoria en el Perú: entre la participación y la gobernabilidad local. En F. Tuesta (ed.), Una onda expansiva. Las revocatorias en el Perú y América Latina (pp. 45-66). Lima, Perú: Jurado Nacional de Elecciones, Pontificia Universidad Católica del Perú.

Uribe, C. (2016). La activación de la revocatoria de mandato en el ámbito municipal en Colombia. Lecciones del caso de Bogotá. Estudios Políticos, (48), 179-200.

Vásquez Oruna, E. M. (2014). Las pretensiones revocadoras: el caso de Lima. En F. Tuesta (ed.), Una onda expansiva. Las revocatorias en el Perú y América Latina. Lima: Jurado Nacional de Elecciones (pp. 67-98). Lima, Perú: Pontificia Universidad Católica del Perú.

Vásquez Oruna, E. M. (2014a). Cuando los vientos revocadores azotaron Lima. En Y. Welp y U. Serdült (coords.), La dosis hace el veneno. Análisis de la revocatoria del mandato en América Latina, Estados Unidos y Suiza (pp. 31-56). Quito, Ecuador: Consejo Nacional Electoral República Ecuador, Instituto de la Democracia.

Verdugo, J. (2014a). La inauguración fallida de la revocatoria en Bolivia: una delimitación conceptual. En F. Tuesta (ed.), Una onda expansiva. Las revocatorias en el Perú y América Latina (pp. 187207). Lima, Perú: Jurado Nacional de Elecciones, Pontificia Universidad Católica del Perú.

Verdugo, Julio (2014). Bolivia: entre las expectativas de uso y los intentos fallidos de activación. En Y. Welp y U. Serdült (coords.), La dosis hace el veneno. Análisis de la revocatoria del mandato en América Latina, Estados Unidos y Suiza (pp. 135-158). Quito, Ecuador: Consejo Nacional Electoral República Ecuador, Instituto de la Democracia.

Welp, Y. (2013). ¿Por qué Perú? Análisis de la revocatoria del mandato en perspectiva comparada. Elecciones, 12(13), 53-77.

Welp, Y. (2014). La revocatoria de mandato en la encrucijada. Mecanismos de democracia directa, participación, representación y democracia. En F. Tuesta Soldevilla (ed.), Una onda expansiva. 
Las revocatorias en el Perú y América Latina (pp. 23-43). Lima, Perú: Jurado Nacional de Elecciones, Pontificia Universidad Católica del Perú.

Welp, Y. (2015). Recall referendums in Peruvian municipalities: a political weapon for bad losers or an instrument of accountability? Democratization, 22(7), 1-21. doi: $10.1080 / 13510347.2015 .1060222$

Welp, Y. y Serdült, U. (2011). ¿Jaque a la representación? Análisis de la revocatoria de mandato en los gobiernos locales de América Latina. En Y. Welp y L. Whitehead (comps.), Caleidoscopio de la innovación democrática en América Latina (pp. 145-169). Ciudad de México, México: FLACSO México, Nuffield College, Centre for Research on Direct Democracy.

Welp, Y. y Serdült, U. (2012). ¿Renovación, crisis o más de lo mismo? La revocatoria de mandato en los gobiernos locales latinoamericanos. Desafíos, 24(2), 169-192.

Welp, Y. y Serdült, U. (2014). Cuando es peor el remedio que la enfermedad. Análisis de la revocatoria del mandato en los municipios de los países andinos. En A. Lissidini, Y. Welp y D. Zovatto (comps.), Democracias en movimiento. Mecanismos de democracia directa y participativa en América Latina (pp. 107-129). Ciudad de México, México: Instituto de Investigaciones Jurídicas.

Welp, Y. y Serdült, U. (2017). The levelling up of a political institution. Perspectives on the recall referendum. En S. Ruth, Y. Welp y L. Whitehead (eds.), Let the people rule? Direct democracy in the twenty-first century (pp. 137-154). Colchester, Reino Unido: ECPR Press.

Whitehead, L. (2011). Prólogo. En Y. Welp y L. Whitehead (comps.), Caleidoscopio de la innovación democrática en América Latina (pp. 15-22). Ciudad de México, México: FLACSO México, Nuffield College, Centre for Research on Direct Democracy.

Wiener, E. (2004). Municipios, poder y nuevas élites locales: el caso de las revocatorias en los distritos de Julcamarca y Congalla. Lima, Perú: Sepia.

\section{Fuentes}

IPSOS (2010). Opinión data. Resumen de Encuestas a la opinión pública. Año 10, № 129.

IPSOS (2013). Opinión data. Resumen de Encuestas a la opinión pública. Año 13, № 165.

IPSOS-APOYO (2012). Opinión data. Resumen de Encuestas a la opinión pública. Año 12, $\mathrm{N}^{\circ}$ 159.

Ley $\mathrm{N}^{\circ} 8102$. Ley orgánica de municipios de Córdoba. Fiscalía de Estado. Dirección de Informática Jurídica, Córdoba, Argentina 15 de noviembre, 1991. Disponible en línea en: http://tododeiure.atspace.com/leyes/cordoba/8102.htm

Ley $\mathrm{N}^{\circ}$ 357. Legislatura de la Ciudad Autónoma de Buenos Aires (2000). Boletín Oficial de la Ciudad Autónoma de Buenos Aires No 942, Buenos Aires, Argentina, 30 de marzo, 2000.

Disponible online en: http://www.cedom.gov.ar/es/legislacion/normas/leyes/ley357.html

Estado plurinacional de Bolivia (2009). Constitución Política.

Ley $N^{\circ}$ 026. Ley del régimen electoral. Gaceta Oficial de Bolivia, La Paz, Estado Plurinacional de

Bolivia, 30 de junio, 2010.

República del Ecuador (1998). Constitución Política.

República del Ecuador (2008). Constitución Política.

República del Ecuador (2010). Ley orgánica de participación ciudadana.

Ley 131 por la cual se reglamenta el voto programático y se dictan otras disposiciones. Diario

Oficial N 41.351, Santa Fe de Bogotá, D.C., República de Colombia, 9 de mayo, 1994.

Ley 134, sobre mecanismos de participación ciudadana. Diario Oficial $N^{\circ} 41.373$, Santa Fe de Bogotá, D.C., República de Colombia, 31 de mayo, 1994.

Ley 741, por la cual se reforman las Leyes 131 y 134 de 1994. Diario Oficial $N^{\circ} 44.823$, Bogotá, D.C., República de Colombia, 4 de junio, 2002. 
Ley 30315. Ley que modifica diversos artículos de la Ley 26300, Ley de Derechos de Participación y Control Ciudadano. El Peruano, Lima, Perú, 13 de marzo, 2015.

Ley 26300, Ley de Derechos de Participación y Control Ciudadano El Peruano, Lima, Perú, 2 de mayo, 1994.

República Bolivariana de Venezuela (1999). Constitución Política.

Resolución $N^{\circ}$. 030925-465. Normas para regular los procesos de referendos revocatorias de mandato de cargos de elección popular. Gaceta Oficial No 37.784, Caracas, República Bolivariana de Venezuela, 25 de septiembre, 2003.

Resolución N. 070207-036. Gaceta Oficial N³56, Caracas, República Bolivariana de Venezuela, 12 de febrero, 2007.

\section{Medios de prensa escrita}

Bottinelli, A. (1 de febrero de 2005).El alcalde de Buenos Aires somete su cargo a referéndum tras incendio. La Voz de Galicia, Buenos Aires. Recuperado de http://www.lavozdegalicia.es/internacional/2005/02/01/0003_3422898.htm.

Clarín (4 de diciembre de 2003). El intendente cordobés Germán Kammerath fue imputado por presunta administración fraudulenta. Clarín. Recuperado de https://www.clarin.com/ultimomomento/intendente-cordobes-german-kammerath-imputado-presunta-administracion-fraudulenta_0_BJ2zvg1eCFl.html

El pais (11 de febrero de 2014). "Pacho" Santos lidera campaña por la revocatoria de Petro. El País. Recuperado de http://www.elpais.com.co/elpais/colombia/noticias/ pacho-santos-lidera-campana-por-revocatoria-petro.

Infobae (13 de marzo de 2014). Avanza una campaña de recolección de firmas para revocar el mandato de Macri. Infobae. Recuperado de http://www.infobae.com/2014/03/13/1549811avanza-una-campana-recoleccion-firmas-revocar-el-mandato-macri.

La Hora (27 de agosto de 2010). Piden revocatoria al mandato del Alcalde. La Hora, Quito. Recuperado de http://lahora.com.ec/index.php/noticias/show/1101009067/-1/Piden_revocatoria_al_mandato_del_Alcalde.html.

Lancheros, Y. (4 de diciembre de 2012). Revocatorias de alcaldes en Colombia nunca han tenido éxito. El Tiempo. Recuperado de http://www.eltiempo.com/archivo/documento/ CMS-12421266.

Mestanza, J. C. et al. (7 de julio de 2015). La mayoría de pedidos para revocar el mandato a autoridades fue archivada. El Comercio. Recuperado de http://www.elcomercio.com/actualidad/ revocatoria-mandato-autoridades-ecuador-politica.html.

Redacción El Tiempo (16 de septiembre de 1999a). La revocatoria del mandato se hunde mandato se hundió. El Tiempo. Recuperado de http://www.eltiempo.com/archivo/documento/ MAM-891221.

Redacción El Tiempo (5 de junio de 1999b). Vigilancia a revocatoria de mandato. El Tiempo. Recuperado de http://www.eltiempo.com/archivo/documento/MAM-893575.

Redacción LR (15 de marzo de 2013). Audios revelan que Luis Castañeda está detrás de la revocatoria de Susana Villarán. La República. Recuperado de http://larepublica.pe/15-03-2013/ audios-revelan-que-luis-castaneda-esta-detras-de-la-revocatoria-de-susana-villaran

Semana (3 de enero de 2000). Enrique Peñalosa. Semana. Recuperado de http://www.semana.com/ nacion/articulo/enrique-penalosa/40470-3. 